TITLE:

\title{
Canonical functions, differential graded symplectic pairs in supergeometry, and Alexandrov- Kontsevich-Schwartz-Zaboronsky sigma models with boundaries
}

\section{$\operatorname{AUTHOR}(\mathrm{S}):$}

Ikeda, Noriaki; Xu, Xiaomeng

\section{CITATION:}

Ikeda, Noriaki ... [et al]. Canonical functions, differential graded symplectic pairs in supergeometry, and Alexandrov-Kontsevich-Schwartz-Zaboronsky sigma models with boundaries. Journal of Mathematical Physics 2014, 55(11): 113505.

\section{ISSUE DATE:}

2014-11

URL:

http://hdl.handle.net/2433/192442

\section{RIGHT:}

Copyright 2014 American Institute of Physics. This article may be downloaded for personal use only. Any other use requires prior permission of the author and the American Institute of Physics. 


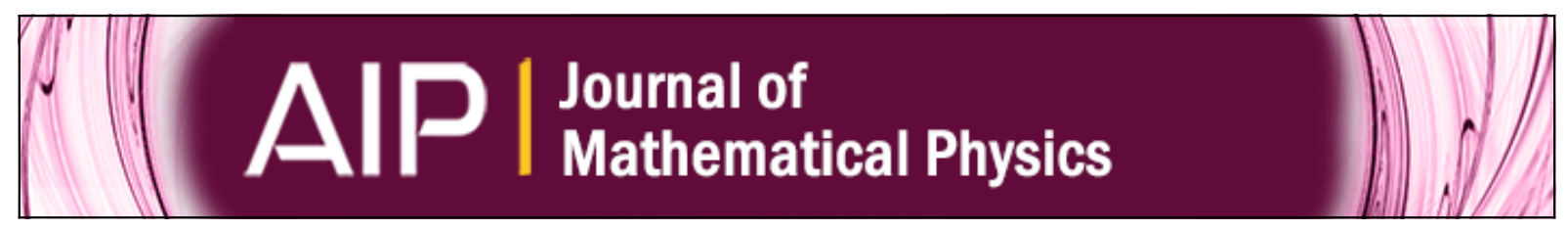

Canonical functions, differential graded symplectic pairs in supergeometry, and Alexandrov-Kontsevich-Schwartz-Zaboronsky sigma models with boundaries

Noriaki Ikeda and Xiaomeng $\mathrm{Xu}$

Citation: Journal of Mathematical Physics 55, 113505 (2014); doi: 10.1063/1.4900834

View online: http://dx.doi.org/10.1063/1.4900834

View Table of Contents: http://scitation.aip.org/content/aip/journal/jmp/55/11?ver=pdfcov

Published by the AIP Publishing

\section{Articles you may be interested in}

Canonical surfaces associated with projectors in Grassmannian sigma models

J. Math. Phys. 51, 103509 (2010); 10.1063/1.3486690

4Manifolds with a Symplectic Bias

AIP Conf. Proc. 1023, 34 (2008); 10.1063/1.2958177

Superconformal primary fields on a graded Riemann sphere

J. Math. Phys. 45, 2492 (2004); 10.1063/1.1739295

Publisher's Note: "On certain geometric aspects of CP N harmonic maps" [J. Math. Phys. 44, 813 (2003)]

J. Math. Phys. 44, 2723 (2003); 10.1063/1.1576171

Kähler potential of moduli space of Calabi-Yau d-fold embedded in CP d+1

J. Math. Phys. 41, 8339 (2000); 10.1063/1.1319848

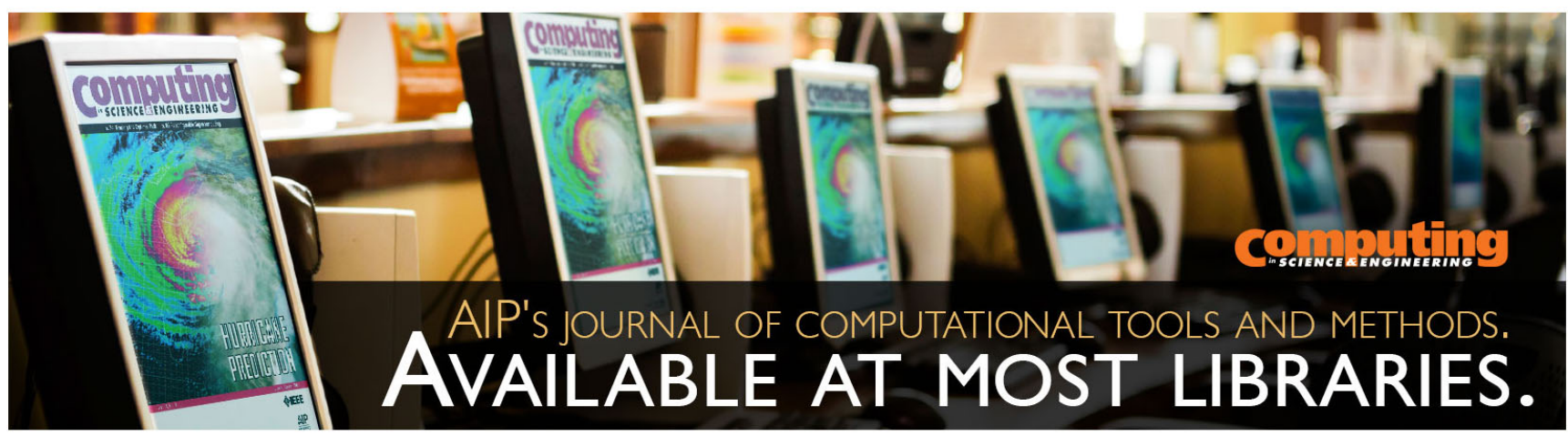




\title{
Canonical functions, differential graded symplectic pairs in supergeometry, and Alexandrov-Kontsevich- Schwartz-Zaboronsky sigma models with boundaries
}

\author{
Noriaki Ikeda ${ }^{1, a)}$ and Xiaomeng $\mathrm{Xu}^{2, \mathrm{~b})}$ \\ ${ }^{1}$ Department of Mathematical Sciences, Ritsumeikan University, Kusatsu, Shiga 525-8577, \\ Japan and Maskawa Institute for Science and Culture, Kyoto Sangyo University, \\ Kyoto 603-8555, Japan \\ ${ }^{2}$ Section of Mathematics, University of Geneva, 2-4 Rue de Lièvre, c. p. 64, 1211-Genève 4, \\ Switzerland
}

(Received 25 February 2014; accepted 20 October 2014; published online 11 November 2014)

\begin{abstract}
Consistent boundary conditions for Alexandrov-Kontsevich-Schwartz-Zaboronsky (AKSZ) sigma models and the corresponding boundary theories are analyzed. As their mathematical structures, we introduce a generalization of differential graded symplectic manifolds, called twisted QP manifolds, in terms of graded symplectic geometry, canonical functions, and QP pairs. We generalize the AKSZ construction of topological sigma models to sigma models with Wess-Zumino terms and show that all the twisted Poisson-like structures known in the literature can actually be naturally realized as boundary conditions for AKSZ sigma models. ( 2014 AIP Publishing LLC. [http://dx.doi.org/10.1063/1.4900834]
\end{abstract}

\section{INTRODUCTION}

A differential graded symplectic manifold (QP manifold) has been introduced from analysis of the Batalin-Vilkovisky formalism. ${ }^{43}$ It was later used for a simple procedure for constructing topological sigma models by Alexandrov, Kontsevich, Schwarz, and Zaboronsky. ${ }^{2}$ Following this, graded symplectic geometry has been of interest in both mathematics and physics due to its rich mathematical structures and links with a variety of topics; see Refs. 11,39, 47, and 48.

A Poisson manifold is naturally a QP manifold of degree 1. A Courant algebroid, which was introduced in Ref. 35 to describe the double of Lie bialgebroids, has a one-to-one correspondence to a QP manifold of degree 2. ${ }^{39}$ The corresponding Alexandrov-Kontsevich-Schwartz-Zaboronsky (AKSZ) sigma models are the Poisson sigma model ${ }^{23,26,42}$ and the Courant sigma model, ${ }^{24,41}$ respectively. Weaker versions of Poisson structures and variants of Lie algebroids and Courant algebroids are motivated by questions from quantum groups and field theories. For example, an extension of the Poisson sigma model by a Wess-Zumino (WZ) term naturally leads to WZ-Poisson manifolds, ${ }^{29,38}$ or equivalently, the Poisson structures with a 3 -form background. ${ }^{44} \mathrm{~A}$ similar structure was studied in the framework of the Courant algebroid theory. Hansen and Strobl ${ }^{20}$ showed that a generalization of Courant algebroids with a 4-form arises naturally in the Courant sigma model with a Wess-Zumino term.

Our main result shows that all the twisted Poisson-like structures known in the literature can actually be naturally realized as (and so in a certain sense "are") boundary conditions for AKSZ sigma models. This result greatly clarifies the meaning of the otherwise rather obscure conditions defining a twisted Poisson-like structure.

To do this, we introduce a canonical function and a QP pair (a differential graded symplectic pair), which are generalizations of differential graded structures in graded symplectic geometry. This

\footnotetext{
a)Electronic addresses: nikeda@se.ritsumei.ac.jp and ikeda@yukawa.kyoto-u.ac.jp

b)E-mail: Xiaomeng.Xu@unige.ch
} 
idea is inspired by analysis of the consistency of boundary conditions of AKSZ sigma models, 2,8,25,41 which are topological sigma models constructed by supergeometric methods and a topological open membrane. ${ }^{22,38}$ A similar structure appears in the Batalin-Vilkovisky (BV) formalism of string field theory. $^{21}$

Another motivation comes from the canonical transformation in symplectic supergeometry, which is also called twisting. ${ }^{40}$ It can be viewed as a higher analogue of a Poisson function, ${ }^{33,45}$ and it defines a generalization of the Dirac structure ${ }^{12}$. Moreover, a canonical function describes the boundary condition structures of the AKSZ sigma models, which have played key roles in the derivation of the deformation quantization from the Poisson sigma model. ${ }^{30}$ and ${ }^{7}$

A canonical function leads to the concept of a QP pair, which is a certain tower of two (twisted) differential graded symplectic manifolds. This unifies the various concepts that were separately analyzed above, and it includes many geometric structures such as the Lie (2-)algebras, the (twisted or quasi) Poisson structures, the (homotopy) Lie algebroids, the (twisted) Courant algebroids, the Nambu-Poisson structures, and others. Some of these will be used below as examples.

Analysis of a canonical transformation on a QP manifold naturally leads to what we call a twisted QP manifold. It is a mathematical framework for a unified understanding of the so-called Wess-Zumino terms, together with twisted Poisson-like structures. A general method to get a twisted QP manifold is given by the deformation theory. Some examples will be presented to illuminate this twisting process. Moreover a new geometric structure, the strong Courant algebroid, is proposed.

The defining structure of a QP pair guarantees consistency of the bulk structure and the boundary conditions. In general, a quantum theory on a manifold $X$ in $n+1$ dimensions with given boundaries may have the same structure as the corresponding quantum theory on the boundary $\partial X$ in $n$ dimensions. ${ }^{3}$ When applying this so-called bulk-boundary correspondence of quantum field theories to the AKSZ sigma models, we find that it is necessary to extend the AKSZ sigma models to the "twisted" AKSZ sigma models. This is a generalization of the Chern-Simons/WZW correspondence. We propose an extension of the AKSZ construction to a twisted version via a map from twisted QP manifolds to topological sigma models.

There are recent works on similar topics. Twisted structures in the setting of $L_{\infty}$-algebras are analyzed in Refs. 17 and 18. There are studies that present formulations of AKSZ sigma models with boundaries. ${ }^{9,10,15,16,28}$ The concept of QP pairs is used in the current algebra theory in Ref. 28. In Ref. 34, the twisted QP manifold introduced in this paper is shown to be a homotopy version of a QP manifold in the spirit of a homotopy Poisson manifold. The AKSZ sigma models have been reformulated by using derived geometry. 5,37

This paper is organized as follows. In Sec. II, we review a QP manifold and an AKSZ sigma model without boundaries. In Sec. III, we analyze the AKSZ sigma models with boundaries and introduce canonical functions. In Sec. IV, we analyze a twisted QP manifold and present some examples. In particular, we propose a new algebroid, the (twisted) strong Courant algebroid. In Sec. V, we consider a physical application of a twisted QP manifold whose defining structure guarantees consistency of the corresponding AKSZ sigma models with boundaries. Bulkboundary correspondence of sigma models leads us to the concept of twisted AKSZ sigma models. Section VI summarizes our results and considers areas of future work.

\section{QP MANIFOLDS AND AKSZ SIGMA MODELS}

\section{A. QP manifolds}

A graded manifold $\mathcal{M}$ is a ringed space with a structure sheaf of a bZ-graded commutative algebra over an ordinary smooth manifold $M$. Grading is compatible with supermanifold grading, that is, a variable of even degree is commutative, and one of odd degree is anticommutative. By definition, the structure sheaf of $\mathcal{M}$ is locally isomorphic to $C^{\infty}(U) \otimes S^{\bullet}(V)$, where $U$ is a local chart on $M, V$ is a graded vector space, and $S^{\bullet}(V)$ is a free graded commutative ring on $V$. Refer to Refs. 6 and 46 for the rigorous mathematical definition of objects in supergeometry.

An N-manifold (i.e., a nonnegatively graded manifold) $\mathcal{M}$ equipped with a graded symplectic structure $\omega$ of degree $n$ is called a $P$-manifold of degree $n$, denoted by $(\mathcal{M}, \omega) . \omega$ is also called a 
$P$-structure. A graded Poisson bracket on $C^{\infty}(\mathcal{M})$ is defined as $\{f, g\}=(-1)^{|f|-n+1} i_{X_{f}} i_{X_{g}} \omega$, where a Hamiltonian vector field $X_{f}$ is defined by the equation $i_{X_{f}} \omega=-\delta f$ for any $f \in C^{\infty}(\mathcal{M})$, and $\delta$ is a differential on $\mathcal{M}$. A vector field $Q$ on $\mathcal{M}$ is called homological if $Q^{2}=0$.

Definition 2.1. A QP-manifold is a P-manifold $(\mathcal{M}, \omega)$ endowed with a degree 1 homological vector field $Q$ such that $\mathcal{L}_{Q} \omega=0 .{ }^{43}$

We call the homological vector field $Q$ the Q-structure. We also denote a $\mathrm{QP}$ manifold by the corresponding triple $(\mathcal{M}, \omega, Q)$. For any QP manifold of positive degree, there exists a Hamiltonian function $\Theta \in C^{\infty}(\mathcal{M})$ of $Q$ with respect to the graded Poisson bracket $\{-,-\}$, that is,

$$
Q=\{\Theta,-\} .
$$

Then, the homological condition, $Q^{2}=0$, implies that $\Theta$ is a solution of the classical master equation,

$$
\{\Theta, \Theta\}=0 .
$$

Such a $\Theta$ is also called a homological function.

\section{B. AKSZ sigma models without boundaries}

In this subsection, we review the AKSZ construction, ${ }^{2,8,41}$ which is a systematic method for constructing a topological sigma model from a QP manifold. The resulting sigma model is called an AKSZ sigma model.

Let $(\mathcal{X}, D, \mu)$ be a differential graded manifold $\mathcal{X}$ with a $D$-invariant nondegenerate measure $\mu$, where $D$ is a differential on $\mathcal{X}$. Let $(\mathcal{M}, \omega, Q)$ be a QP-manifold, and let $\operatorname{Map}(\mathcal{X}, \mathcal{M})$ be the space of smooth maps from $\mathcal{X}$ to $\mathcal{M}$.

Since $\operatorname{Diff}(\mathcal{X}) \times \operatorname{Diff}(\mathcal{M})$ naturally acts on $\operatorname{Map}(\mathcal{X}, \mathcal{M}), D$ and $Q$ induce differentials $\hat{D}$ and $\hat{Q}$, respectively, on $\operatorname{Map}(\mathcal{X}, \mathcal{M})$. Explicitly, $\hat{D}(z, f)=\delta f(z) D(z)$ and $\mathscr{Q}(z, f)=Q f(z)$, for all $z \in \mathcal{X}$ and $f \in \operatorname{Map}(\mathcal{X}, \mathcal{M})$.

An evaluation map ev : $\mathcal{X} \times \operatorname{Map}(\mathcal{X}, \mathcal{M}) \longrightarrow \mathcal{M}$ is defined as ev $:(z, f) \longmapsto f(z)$, for any $z \in \mathcal{X}$ and $f \in \operatorname{Map}(\mathcal{X}, \mathcal{M})$. A chain map $\mu_{*}: \Omega^{\bullet}(\mathcal{X} \times \operatorname{Map}(\mathcal{X}, \mathcal{M})) \longrightarrow \Omega^{\bullet}(\operatorname{Map}(\mathcal{X}, \mathcal{M}))$ is defined as

$$
\mu_{*} \omega(y)\left(v_{1}, \ldots, v_{k}\right)=\int_{\mathcal{X}} \mu(x) \omega(x, y)\left(v_{1}, \ldots, v_{k}\right),
$$

where $v$ is a vector field on $\mathcal{X}$ and $\int_{\mathcal{X}} \mu$ is the Berezin integration on $\mathcal{X}$. The composition $\mu_{*} \mathrm{ev}^{*}$ : $\Omega^{\bullet}(\mathcal{M}) \longrightarrow \Omega^{\bullet}(\operatorname{Map}(\mathcal{X}, \mathcal{M}))$ is called the transgression map.

A P-structure $\omega$ (a graded symplectic structure) on $\operatorname{Map}(\mathcal{X}, \mathcal{M})$ is defined by

$$
\omega:=\mu_{*} \mathrm{ev}^{*} \omega .
$$

Note that $\omega$ is nondegenerate and closed because the operation $\mu_{*} \mathrm{ev}^{*}$ preserves these properties. The corresponding graded Poisson bracket on $\operatorname{Map}(\mathcal{X}, \mathcal{M})$ is denoted by $\{-,-\}$.

A Q-structure function $S$ on $\operatorname{Map}(\mathcal{X}, \mathcal{M})$ is constructed as follows. $S$ consists of two parts $S$ $:=S_{0}+S_{1}$. We take a canonical 1-form $\vartheta$ on $\mathcal{M}$ such that $\omega=-\delta \vartheta$ and define $S_{0}:=\iota_{\hat{D}} \mu_{*} \mathrm{ev}^{*} \vartheta$. Moreover, we define $S_{1}:=\mu_{*} \mathrm{ev}^{*} \Theta$, where $\Theta$ is the homological function on $\mathcal{M}$. Then we can prove that $S$ is a homological function on $\operatorname{Map}(\mathcal{X}, \mathcal{M})$,

$$
\{S, S\}=0,
$$

from the definitions of $S_{0}$ and $S_{1}$ and the properties of maps. A degree 1 homological vector field $\boldsymbol{Q}$ is defined as $\boldsymbol{Q}=\{S,-\}$. The classical master equation shows that $\boldsymbol{Q}$ is a coboundary operator, $Q^{2}=0$.

We thus have the following theorem.

Theorem 2.2. (Ref. 2). Let $\mathcal{X}$ be a differential graded manifold with a compatible measure, and let $\mathcal{M}$ be a $Q P$-manifold, then the mapping space $\operatorname{Map}(\mathcal{X}, \mathcal{M})$ has a QP structure. 
This structure is called an AKSZ sigma model. If $\mathcal{X}=T[1] X$, where $X$ is a manifold in $n+$ 1 dimensions, the QP structure on $\operatorname{Map}(\mathcal{X}, \mathcal{M})$ is of degree -1 . In this case, a QP structure on $\operatorname{Map}(T[1] X, \mathcal{M})$ is equivalent to the Batalin-Vilkovisky formalism of a topological sigma model.

\section{AKSZ SIGMA MODELS WITH BOUNDARIES}

In this section, the geometric structure of the AKSZ sigma model on a base manifold $X$ with a boundary is analyzed and proven to be described by a quintuple mathematical datum, $(\mathcal{M}, \omega, \Theta, \mathcal{L}, \alpha)$. When $n=1$, this corresponds to a topological open string and produces a deformation quantization formula. ${ }^{7}$ When $n \geq 2$, such theories describe topological open membranes. ${ }^{38,22}$

\section{A. Consistent boundary conditions}

Let us take a manifold $X$ in $n+1$ dimensions with nonempty boundaries. Let $(\mathcal{X}=$ $T[1] X, D, \mu)$ be a differential graded manifold over $X$ with a differential $D$ and a compatible measure $\mu$, and let $(\mathcal{M}, \omega, \Theta)$ be a QP manifold of degree $n$. Then the AKSZ construction produces a consistent topological sigma model on the mapping space $\operatorname{Map}(T[1] X, \mathcal{M})$, as long as the boundary conditions on $\partial \mathcal{X}$ are consistent with the QP structure of the whole theory. Generally the classical master equation is not satisfied because of the boundary terms. By Stokes' theorem, a straightforward computation gives

$$
\{S, S\}=\int_{\partial \mathcal{X}} \mu_{\partial \mathcal{X}}\left(i_{\partial} \times \mathrm{id}\right)^{*} \operatorname{ev}^{*}(\vartheta+\Theta),
$$

where $\mu_{\partial \mathcal{X}}$ is a boundary measure induced from $\mu$ to $\partial \mathcal{X}$ by the inclusion map $i_{\partial}: \partial \mathcal{X} \longrightarrow \mathcal{X}$. Since the classical master equation $\{S, S\}=0$ must be satisfied for consistency of the theory, the right-hand side of Eq. (3.1) must vanish. From this observation, we have

Theorem 3.1. If $\left.(\vartheta+\Theta)\right|_{\operatorname{Im} \partial \mathcal{X}}=0$ on $\mathcal{M}$, then $\{S, S\}=0$.

Let us consider the physical constraints. $S$ is a classical BV action in a physical theory. In order to derive the equation of motion from the variational principle in mechanics, the variation of $S$ must vanish on the boundaries. This is satisfied if $\vartheta=0$ on $\operatorname{Im} \partial \mathcal{X}$. Since $\omega=-\delta \vartheta$, this says that $\operatorname{Im} \partial \mathcal{X}$ belongs to a subspace of a Lagrangian submanifold $\mathcal{L} \subset \mathcal{M}$ that is the zero locus of $\vartheta=0$. This physical requirement turns Theorem 3.1 into the following condition on $\mathcal{M}$ :

Proposition 3.2. Let $\mathcal{L}$ be a Lagrangian submanifold of $\mathcal{M}$, i.e., $\left.\vartheta\right|_{\mathcal{L}}=0$. Then $\{S, S\}=0$ is satisfied if $\left.\Theta\right|_{\mathcal{L}}=0$.

We demonstrate that this is consistent with the variational principle by taking the Darboux coordinates of the superfields with respect to the $\mathrm{P}$-structure $\omega$ on $\operatorname{Map}(\mathcal{X}, \mathcal{M})$, where the superfields are pullbacks of the local coordinates on $\mathcal{M}$ by $\boldsymbol{x}^{*}$, the degree zero map $\boldsymbol{x}: \mathcal{X} \longrightarrow M$. We denote $\boldsymbol{q}^{a(i)}(\sigma, \theta) \in \Gamma\left(T[1] X \otimes \boldsymbol{x}^{*}\left(\mathcal{M}_{i}\right)\right)$ for $0 \leq i \leq\lfloor n / 2\rfloor$ and $\boldsymbol{p}_{a(n-i)}(\sigma, \theta) \in \Gamma\left(T[1] X \otimes \boldsymbol{x}^{*}\left(\mathcal{M}_{n-i}\right)\right)$ for $\lfloor n / 2\rfloor<i \leq n$, where $(\sigma, \theta)$ are local coordinates on $\mathcal{X}=T[1] X, \mathcal{M}_{i}$ is the degree $i$ subspace of $\mathcal{M}, \boldsymbol{x}=\boldsymbol{q}^{a(0)}$, and $\lfloor m\rfloor$ is the floor function, which gives the largest integer less than or equal to $m$. The Poisson brackets of superfields are

$$
\left\{\boldsymbol{q}^{a(i)}(\sigma, \theta), \boldsymbol{p}_{b(j)}\left(\sigma^{\prime}, \theta^{\prime}\right)\right\}=\delta^{i}{ }_{j} \delta^{a(i)}{ }_{b(j)} \delta^{n+1}\left(\sigma-\sigma^{\prime}\right) \delta^{n+1}\left(\theta-\theta^{\prime}\right),
$$

and if $n$ is even and $i=j=n / 2$,

$$
\left\{\boldsymbol{q}^{a(n / 2)}(\sigma, \theta), \boldsymbol{q}^{b(n / 2)}\left(\sigma^{\prime}, \theta^{\prime}\right)\right\}=k^{a(n / 2) b(n / 2)} \delta^{n+1}\left(\sigma-\sigma^{\prime}\right) \delta^{n+1}\left(\theta-\theta^{\prime}\right),
$$

where $k^{a(n / 2) b(n / 2)}$ is a metric on $\mathcal{M}_{n / 2}$. The exterior derivative $d$ on $X$ induces a superdifferential $\boldsymbol{d}=\theta^{\mu} \frac{\partial}{\partial \sigma^{\mu}}$ on $\operatorname{Map}(\mathcal{X}, \mathcal{M})$. If we define $\boldsymbol{p}_{a(n / 2)}=k_{a(n / 2) b(n / 2)} \boldsymbol{q}^{a(n / 2)}$, then $S$ takes the same form for 
odd or even $n$,

$$
S=S_{0}+S_{1}=\int_{\mathcal{X}} \mu\left(\sum_{0 \leq i \leq\lfloor n / 2\rfloor}(-1)^{n+1-i} \boldsymbol{p}_{a(i)} \boldsymbol{d} \boldsymbol{q}^{a(i)}\right)+\int_{\mathcal{X}} \mu \operatorname{ev}^{*} \Theta(\boldsymbol{q}, \boldsymbol{p}) .
$$

In order to derive the equations of motion of the superfields, the variation of $S=S_{0}+S_{1}$ must vanish on the boundaries. The variation is $\delta S=\int_{\partial \mathcal{X}} \mu_{\partial \mathcal{X}} \sum_{0 \leq i \leq(n-1) / 2}(-1)^{n+1-i} \boldsymbol{p}_{a(i)} \delta \boldsymbol{q}^{a(i)}$ up to the equations of motion. Thus, we should impose the condition $\boldsymbol{p}_{a(i)} \delta \boldsymbol{q}^{a(i)}=0$ on the boundary $\partial \mathcal{X}$.

\section{B. Canonical transformations and canonical functions}

A physical theory can have boundary terms if the base manifold $X$ has a boundary. We introduce boundary terms by a canonical transformation.

Let $(\mathcal{M}, \omega, Q)$ be a QP manifold of degree $n$, and suppose that $Q$ is generated by a Hamiltonian function $\Theta \in C^{\infty}(\mathcal{M})$ of degree $n+1$. Let $\alpha \in C^{\infty}(\mathcal{M})$. We define an exponential operation $e^{\delta_{\alpha}}$ by

$$
e^{\delta_{\alpha}} f=f+\{f, \alpha\}+\frac{1}{2}\{\{f, \alpha\}, \alpha\}+\cdots,
$$

for any $f \in C^{\infty}(\mathcal{M})$.

Definition 3.3. For any function $\alpha$ of degree $n, e^{\delta_{\alpha}}$ is called a canonical transformation.

Note that $e^{\delta_{\alpha}}$ was called twisting in Ref. 40. In addition, for any $\alpha$ of degree $n,\left\{e^{\delta_{\alpha}} f, e^{\delta_{\alpha}} g\right\}=$ $e^{\delta_{\alpha}}\{f, g\}$, where $f, g \in C^{\infty}(\mathcal{M})$. Now we introduce the canonical function.

Definition 3.4. Let $(\mathcal{M}, \omega, Q=\{\Theta,-\})$ be a $Q P$ manifold of degree $n$. A function $\alpha$ of degree $n$ is called a canonical function with respect to $\mathcal{L}$ if $\left.e^{\delta_{\alpha}} \Theta\right|_{\mathcal{L}}=0$, where $\mathcal{L}$ is a Lagrangian submanifold of $(\mathcal{M}, \omega)$ and $\left.\right|_{\mathcal{L}}$ is the restriction on $\mathcal{L}$.

Obviously if $\alpha$ is a canonical function on a QP manifold $(\mathcal{M}, \omega, \Theta), e^{\delta_{\alpha}}$ is a canonical transformation.

We make a canonical transformation $e^{\delta_{\alpha}}$ by a function $\alpha$ of degree $n$. This changes a target QP manifold to $\left(\mathcal{M}, \omega, \Theta_{\alpha}\right)$, where the Q-structure is $\Theta_{\alpha}=e^{\delta_{\alpha}} \Theta$. Since the P-structure does not change, a new Q-structure $S^{\prime}$ on $\operatorname{Map}(T[1] X, \mathcal{M})$ in the AKSZ sigma model becomes

$$
\begin{aligned}
S^{\prime} & =S_{0}+S_{1}^{\prime} \\
& =\iota_{\hat{D}} \mu_{*} \mathrm{ev}^{*} \vartheta+\mu_{*} \mathrm{ev}^{*} e^{\delta_{\alpha}} \Theta .
\end{aligned}
$$

If $\partial X=\emptyset, S^{\prime}$ also satisfies the classical master equation because of $\left\{e^{\delta_{\alpha}} \Theta, e^{\delta_{\alpha}} \Theta\right\}=e^{\delta_{\alpha}}\{\Theta, \Theta\}=0$. If $\partial X \neq \emptyset$, we should modify the boundary conditions so that $S^{\prime}$ satisfies the classical master equation. Applying Proposition 3.2 to Eq. (3.5), we have

Proposition 3.5. (Ref. 22). We assume $\partial \mathcal{X} \neq \emptyset$. Let $(\mathcal{M}, \omega, \Theta)$ be a $Q P$ manifold of degree $n$, and let $\alpha \in C^{\infty}(\mathcal{M})$ of degree $n$ be a canonical transformation. The classical master equation $\{S$, $S\}=0$ is satisfied in an AKSZ sigma model (3.5) if $\left.e^{\delta_{\alpha}} \Theta\right|_{\mathcal{L}}=0$, that is, $\alpha$ is a canonical function with respect to a Lagrangian submanifold $\mathcal{L}$ of $\mathcal{M}$.

From Proposition 3.5, the mathematical structure of an AKSZ sigma model with a boundary is a quintuple $(\mathcal{M}, \omega, \Theta, \mathcal{L}, \alpha)$.

The first three elements of the datum form a QP manifold $(\mathcal{M}, \omega, \Theta)$. The full quintuple is a $\mathrm{QP}$ manifold with a trivialization of $\omega$ and $\Theta$. The trivialization of $\omega,\left.\omega\right|_{\mathcal{L}}=0$ determines a Lagrangian submanifold $\mathcal{L}$ of $\mathcal{M}$ (we take the maximal $\mathcal{L}$ ). Finally, we require a trivialization of $\Theta$, but this can be refined so that $\Theta$ vanishes on $\mathcal{L}$ up to a canonical transformation $e^{\delta_{\alpha}}$, i.e., $\left.e^{\delta_{\alpha}} \Theta\right|_{\mathcal{L}}=0$ such that $|\alpha|=|\omega|$. 


\section{From canonical functions to boundary terms}

We see that $\alpha$ corresponds to physical boundary terms in a special case.

In Eq. (3.5), the geometric structure is equivalent even if we make the inverse canonical transformation on $\operatorname{Map}(\mathcal{X}, \mathcal{M})$ for the P-structure in addition to that for the Q-structure. We then have an equivalent expression for the homological function:

$$
\begin{aligned}
S^{\prime \prime} & =e^{-\delta_{\alpha}} S^{\prime} \\
& =\iota_{\hat{D}} \mu_{*} \mathrm{ev}^{*} e^{-\delta_{\alpha}} \vartheta+\mu_{*} \mathrm{ev}^{*} e^{-\delta_{\alpha}} e^{\delta_{\alpha}} \Theta \\
& =\iota_{\hat{D}} \mu_{*} \mathrm{ev}^{*} e^{-\delta_{\alpha}} \vartheta+\mu_{*} \mathrm{ev}^{*} \Theta .
\end{aligned}
$$

The AKSZ sigma model is $S^{\prime \prime}$, and its geometric structure defines the equivalent QP structure as the original $S^{\prime}$ on $\operatorname{Map}(\mathcal{X}, \mathcal{M})$.

Let us consider the special case such that $\alpha$ satisfies $\{\alpha, \alpha\}=0$. Then, since $e^{-\delta_{\alpha}} \vartheta=\vartheta-\{\vartheta, \alpha\}$, $\alpha$ generates a boundary term $S_{\partial \mathcal{X}}=\mu_{\partial \mathcal{X} *}\left(i_{\partial} \times \mathrm{id}\right)^{*} \operatorname{ev}^{*} \alpha$ by the following computation:

$$
\begin{aligned}
S^{\prime \prime} & =S_{0}-\mu_{*} \operatorname{ev}^{*}\{\vartheta, \alpha\}+\mu_{*} \mathrm{ev}^{*} \Theta \\
& =S_{0}-L_{\hat{D}} \mu_{*} \operatorname{ev}^{*} \alpha+\mu_{*} \operatorname{ev}^{*} \Theta \\
& =\iota_{\hat{D}} \mu_{*} \mathrm{ev}^{*} \vartheta+\mu_{*} \mathrm{ev}^{*} \Theta-\mu_{\partial \mathcal{X} *}\left(i_{\partial} \times \mathrm{id}\right)^{*} \mathrm{ev}^{*} \alpha .
\end{aligned}
$$

Here we have derived this equation from the formula $\left\{S_{0},-\right\}=L_{\hat{D}}(-)$ and the Stokes' theorem, $L_{\hat{D}} \mu_{*} \mathrm{ev}^{*}(-)=\mu_{\partial \mathcal{X}_{*}}\left(i_{\partial}^{*} \times \mathrm{id}\right)^{*} \mathrm{ev}^{*}(-)$, where $L_{\hat{D}}$ is a Lie derivative. Thus a canonical function leads to a boundary source term, $S_{\partial \mathcal{X}}=\mu_{\partial \mathcal{X}_{*}}\left(i_{\partial} \times \mathrm{id}\right)^{*} \mathrm{ev}^{*} \alpha$. Physically, this sigma model describes a topological open membrane with boundary charges. ${ }^{22,38}$ Although general canonical functions can generate more complicated boundary terms which are not integrations of local Lagrangians, Proposition 3.5 says that these also provide physically consistent boundary deformations of the AKSZ sigma models.

In Sec. IV, we will focus on the underlying mathematical structure, a quintuple $(\mathcal{M}, \omega, \Theta, \mathcal{L}, \alpha)$, and a generalization of QP manifolds, twisted QP manifolds, will be introduced and analyzed. Moreover, a new geometric structure, a QP pair, will be proposed.

\section{TWISTED HIGHER-DEGREE POISSON MANIFOLDS}

\section{A. Twisted QP manifolds}

Let $\mathcal{M}$ be a QP manifold of degree $n+1$, and let $\mathcal{L}$ be a Lagrangian submanifold. In this section, we concentrate on the structure on $\mathcal{L}$. By the Lagrangian neighborhood theorem in symplectic geometry, the boundary condition in Proposition 3.5, $(\mathcal{M}, \omega, \Theta, \mathcal{L}, \alpha)$, is locally realized by $\left(T^{*}[n+1] \mathcal{L}, \omega_{c a n}, \Theta, \mathcal{L}, \alpha\right)$. Here $\omega_{\text {can }}$ denotes the canonical symplectic form on the shifted cotangent bundle $T^{*}[n+1] \mathcal{L}$. Therefore, in the following, we will discuss a QP manifold of degree $n+1,\left(T^{*}[n+1] \mathcal{L}, \omega, \Theta\right)$, where $\mathcal{L}$ is an $\mathrm{N}$-manifold of degree $n$. We may assume $\omega=\omega_{\text {can }}$, but this is not necessary.

Let $\pi: T^{*}[n+1] \mathcal{L} \rightarrow \mathcal{L}$ be the natural projection. The derived bracket ${ }^{32}\{-,-\}_{\Theta}$ on $C^{\infty}(\mathcal{L}):$

$$
\{f, g\}_{\Theta}=\left.\left\{\left\{\pi^{*} f, \Theta\right\}, \pi^{*} g\right\}\right|_{\mathcal{L}},
$$

is a graded Poisson bracket for any $f, g \in C^{\infty}(\mathcal{L})$. Because $\{\pi * f, \pi * g\}=0$ and $\{\Theta, \Theta\}=0$, the bracket $\{-,-\}_{\Theta}$ is graded symmetric and satisfies the Leibniz rule and the Jacobi identity. Throughout this section, we assume that the derived bracket is nondegenerate. Then the bracket $\{-,-\}_{\Theta}$ defines a graded symplectic structure $\omega_{\Theta}$ on $\mathcal{L}$.

Let $\alpha_{\mathcal{L}}$ be a function on $\mathcal{L}$ such that $\alpha:=\pi^{*} \alpha_{\mathcal{L}}$ is a canonical function with respect to the Lagrangian submanifold $\mathcal{L}$ of $\left(T^{*}[n+1] \mathcal{L},\{-,-\}, \Theta\right)$. By the definition of canonical functions, we have

$$
\frac{1}{2}\left\{\alpha_{\mathcal{L}}, \alpha_{\mathcal{L}}\right\}_{\Theta}=-\left.\Theta\right|_{\mathcal{L}}-\left.\{\Theta, \alpha\}\right|_{\mathcal{L}}-\left.\frac{1}{3 !}\{\{\{\Theta, \alpha\}, \alpha\}, \alpha\}\right|_{\mathcal{L}}+\cdots
$$


If the right-hand side of this equation is zero, $\left\{\alpha_{\mathcal{L}}, \alpha_{\mathcal{L}}\right\}_{\Theta}=0$. Then $\left(\mathcal{L},\{-,-\}_{\Theta}, \alpha_{\mathcal{L}}\right)$ is a QP manifold of degree $n$. However, in general, the master equation is violated and controlled by the right-hand side of Eq. (4.2), which involves the homological function and the canonical function. This observation leads to the following definition.

Definition 4.1. Let $\left(\mathcal{L},\{-,-\}_{\mathcal{L}}\right)$ be a P-manifold of degree $n$, and let $\alpha_{\mathcal{L}}$ be a degree $n+1$ function on it. Then $\left(\mathcal{L},\{-,-\}_{\mathcal{L}}, \alpha_{\mathcal{L}}\right)$ is called a twisted $Q P$-manifold if there exists a $Q P$-manifold $\left(T^{*}[n+1] \mathcal{L},\{-,-\}, \Theta\right)$ such that

1. $\{f, g\}_{\mathcal{L}}=\left.\left\{\left\{\pi^{*} f, \Theta\right\}, \pi^{*} g\right\}\right|_{\mathcal{L}}$,

2. $\left.e^{\delta_{\alpha}} \Theta\right|_{\mathcal{L}}=0$,

where $\pi: T^{*}[n+1] \mathcal{L} \rightarrow \mathcal{L}$ is the natural projection and $f, g \in C^{\infty}(\mathcal{L})$.

In this case, we call $\left(T^{*}[n+1] \mathcal{L}, \omega, \Theta\right)\left(\mathcal{L}\right.$, sbv-- ${ }_{s}=$ tbv--, $\left.\alpha_{\mathcal{L}}\right)$ the small (twisted) QP manifold, and $\left(T^{*}[n+1] \mathcal{L}, \omega, \Theta, \mathcal{L}\right.$, sbv- $\left.-{ }_{s}, \alpha\right)$ a QP pair, where $\alpha=\pi^{*} \alpha_{\mathcal{L}}$. We also say that $\left(T^{*}[n+1] \mathcal{L}, \omega, \Theta\right)$ is a $\mathrm{QP}$ realization of $\left(\mathcal{L},\{-,-\}_{\mathcal{L}}, \alpha_{\mathcal{L}}\right)$.

The following proposition is an immediate consequence of the definition.

Proposition 4.2. If $\left(\mathcal{L},\{-,-\}_{\mathcal{L}}, \alpha_{\mathcal{L}}\right)$ is a twisted $Q P$ manifold with a $Q P$ realization $\left(T^{*}[n+\right.$ $1] \mathcal{L}, \omega, \Theta)$, then

$$
\frac{1}{2}\left\{\alpha_{\mathcal{L}}, \alpha_{\mathcal{L}}\right\}_{\mathcal{L}}=\frac{1}{2}\left\{\alpha_{\mathcal{L}}, \alpha_{\mathcal{L}}\right\}_{\Theta}=-\left.\Theta\right|_{\mathcal{L}}-\left.\{\Theta, \alpha\}\right|_{\mathcal{L}}-\left.\frac{1}{3 !}\{\{\{\Theta, \alpha\}, \alpha\}, \alpha\}\right|_{\mathcal{L}}-\cdots
$$

In Definition 4.1, it is not clear how to decide whether a given $\left(\mathcal{L},\{-,-\}_{\mathcal{L}}, \alpha_{\mathcal{L}}\right)$ is a twisted QP manifold, since a twisted QP manifold needs extra data $\left(T^{*}[n+1] \mathcal{L}, \omega, \Theta\right)$. Thus a QP pair is a good starting point for the analysis of twisted structures. We consider this problem in Sec. $\mathrm{V}$ in terms of the deformation theory of QP manifolds and the bulk-boundary correspondence of AKSZ sigma models.

\section{B. Examples}

Twisted QP manifolds contain many kinds of twisted higher Poisson structures, for example, the twisted Courant algebroids, Nambu-Poisson structures, and higher twisted algebroids. In this section, we show how known and new structures can be realized as twisted QP manifolds.

First, we give an illustrative example of a canonical function and a twisted QP manifold that is not a QP manifold.

Example 4.1. (Ref. 45). Let $\mathcal{L}=T^{*}[1] M \times \mathfrak{g}[1]$, where $M$ is a manifold and $\mathfrak{g}$ is a quadratic Lie algebra. To define a twisted Q-structure on $\mathcal{L}$, we define a homological function on the shifted cotangent bundle $T^{*}[2] \mathcal{L}$ with a canonical symplectic structure $\omega$. We take local coordinates on $M$, the fiber of $T^{*}[1] M$, and $\mathfrak{g}[1],\left(x^{i}, p_{i}, u^{a}\right)$, respectively. The conjugate coordinates of the fiber of $T^{*}[2]\left(T^{*}[1] M \times \mathfrak{g}[1]\right)$ are $\left(\xi_{i}, q^{i}, v_{a}\right)$. The following function of degree 3 is a homological function on $T^{*}[2] \mathcal{L}$ if $\left\{\Theta_{M}, \Theta_{H}\right\}=0$ and $\left\{\Theta_{C}, \Theta_{R}\right\}=0$ :

$$
\begin{aligned}
\Theta & =\Theta_{M}+\Theta_{C}+\Theta_{R}+\Theta_{H} \\
& =\xi_{i} q^{i}+\frac{1}{2} C_{a b}{ }^{c} u^{a} u^{b} v_{c}+\frac{1}{3 !} R^{a b c} v_{a} v_{b} v_{c}+\frac{1}{3 !} H_{i j k}(x) q^{i} q^{j} q^{k},
\end{aligned}
$$

where $C_{a b}{ }^{c}$ is a Lie algebra structure constant and $R^{a b c}$ is a constant. Let $H$ be a 3 -form on $M$ defined by $H=\frac{1}{3 !} H_{i j k}(x) d x^{i} \wedge d x^{j} \wedge d x^{k}$, and let $R \in \wedge^{3} \mathfrak{g}^{*}$ be defined by $R=\frac{1}{3 !} R^{a b c} v_{a} v_{b} v_{c}$. Note that $\mathfrak{g}, R$ is also seen as a constant section of $\wedge^{3}(M \times \mathfrak{g})$. $\Theta$ is a homological function if and only if $H$ is a closed 3-form on $M$ and $R$ is a Lie algebra 3-cocycle.

Let $\alpha=\pi+\rho=\frac{1}{2} \pi^{i j}(x) p_{i} p_{j}+\rho^{j}{ }_{a}(x) u^{a} p_{j}$ be a canonical function with respect to the Lagrangian submanifold $\mathcal{L}=T^{*}[1] M \times \mathfrak{g}[1]$, which is locally expressed by $\left\{\xi_{i}=q^{i}=v_{a}=0\right\}$. From 
the canonical function equation, a canonical function $\alpha$ satisfies

$$
\frac{1}{2}\left\{\alpha_{\mathcal{L}}, \alpha_{\mathcal{L}}\right\}_{\Theta}=-\left.\frac{1}{3 !}\{\{\{\Theta, \alpha\}, \alpha\}, \alpha\}\right|_{\mathcal{L}} \neq 0 .
$$

Generally, $\alpha$ gives a Lie algebroid structure on $T^{*} M \times \mathfrak{g}$, as discussed in Ref. 36 .

If $\Theta_{C}=\Theta_{R}=\Theta_{H}=0$ and $\rho=0$, the canonical function equation $\left.e^{\delta_{\alpha}} \Theta\right|_{\mathcal{L}}=0$ is equivalent to $\left[\pi_{\mathcal{L}}, \pi_{\mathcal{L}}\right]_{S}=-\left.\{\{\Theta, \pi\}, \pi\}\right|_{\mathcal{L}}=0$, which defines a nontwisted QP manifold and is nothing but a Poisson structure on $M$. Here $[-,-]_{S}=\{-,-\}_{\Theta}$ is identical to the Schouten bracket on $\Gamma\left(\wedge^{\bullet} T M\right)$.

If $\Theta_{H}=0$ and $R$ is the Cartan 3-tensor, then the canonical function equation defines a quasiPoisson structure, $\left[\pi_{\mathcal{L}}, \pi_{\mathcal{L}}\right]_{S}=\wedge^{3} \rho^{\#} R$. ${ }^{1}$

If $\rho=0$, the canonical function equation defines a twisted-Poisson structure, $\left[\pi_{\mathcal{L}}, \pi_{\mathcal{L}}\right]_{S}=$ $\wedge^{3} \pi^{\#} H \cdot{ }^{29,38,44}$ The derived-derived bracket $\left\{\left\{-, \alpha_{\mathcal{L}}\right\}_{\Theta},-\right\}_{\Theta}$ gives a twisted Poisson bracket on $C^{\infty}(M)$.

Example 4.2 ( $\mathrm{H}_{4}$-twisted Courant algebroid). We consider an $\mathrm{N}$-manifold

$T^{*}[3] \mathcal{L}=T^{*}[3] T^{*}[2] E[1]$, where $E \longrightarrow M$ is a vector bundle on a manifold $M$. We take local coordinates $\left(x^{i}, u^{a}, p_{i}\right)$ of degree $(0,1,2)$ on $T^{*}[2] E[1]$ and conjugate local coordinates $\left(\xi_{i}, v_{a}, q^{i}\right)$ of degree $(3,2,1)$ on the fiber.

A graded symplectic structure is given by $\omega=\delta x^{i} \wedge \delta \xi_{i}-\delta u^{a} \wedge \delta v_{a}+\delta p_{i} \wedge \delta q^{i}$ and takes a Lagrangian submanifold $\mathcal{L}=T^{*}[2] E[1]$, which is locally expressed by $\left\{q^{i}=\xi_{i}=v_{a}=0\right\}$.

Let us consider the following Q-structure satisfying $\left.\Theta\right|_{\mathcal{L}}=0$ :

$$
\Theta=\xi_{i} q^{i}+\frac{1}{2} k^{a b} v_{a} v_{b}+\frac{1}{4 !} H_{i j k l}(x) q^{i} q^{j} q^{k} q^{l},
$$

where $k^{a b}$ is a fiber metric on $E$. $\Theta$ satisfies $\{\Theta, \Theta\}=0$ if and only if $d H=0$, where $H=$ $\frac{1}{4 !} H_{i j k l}(x) d x^{i} \wedge d x^{j} \wedge d x^{k} \wedge d x^{l}$ is a 4-form on $M$. This defines a Lie algebroid up to homotopy on $T^{*} E .^{27}$ and ${ }^{19}$

We take the following canonical function of degree 3,

$$
\alpha=\rho_{a}^{i}(x) p_{i} u^{a}+\frac{1}{3 !} h_{a b c}(x) u^{a} u^{b} u^{c},
$$

with respect to the Lagrangian submanifold $\mathcal{L}=T^{*}[2] E[1]$. Straightforward calculations show that the canonical function equation $\left.e^{\delta_{\alpha}} \Theta\right|_{\mathcal{L}}=0$ produces the following identities:

$$
\begin{aligned}
& k^{a b} \rho_{a}^{i} \rho_{b}^{j}=0, \\
& \frac{\partial \rho^{i}{ }_{a}}{\partial x^{j}} \rho^{j}{ }_{b}-\frac{\partial \rho_{b}^{i}}{\partial x^{j}} \rho^{j}{ }_{a}+k^{c d} \rho^{i}{ }_{c} h_{d a b}=0, \\
& \frac{\partial h_{a b c}}{\partial x^{i}} \rho^{i}{ }_{d}+k^{e f} h_{a b e} h_{f c d}+H_{i j k l} \rho^{i}{ }_{a} \rho^{j}{ }_{b} \rho^{k}{ }_{c} \rho^{l}{ }_{d} \\
& \quad+(a b c d \text { completely skewsymmetric })=0 .
\end{aligned}
$$

If $H=0,\left.e^{\delta_{\alpha}} \Theta\right|_{\mathcal{L}}=0$ is equivalent to $\left\{\alpha_{\mathcal{L}}, \alpha_{\mathcal{L}}\right\}_{\Theta}=0$. Then $\left(T^{*}[2] E[1],\{-,-\}_{\Theta}, \alpha_{\mathcal{L}}\right)$ is a QP manifold of degree 2. Equations (4.4), (4.5), and (4.6) are satisfied if and only if $E$ is the Courant algebroid. ${ }^{35,39}$ The Dorfman bracket of the Courant algebroid is given by the derived-derived bracket on $\Gamma(E)$ by

$$
\left[e_{1}, e_{2}\right]_{D}=-\left\{\left\{e_{1}, \alpha_{\mathcal{L}}\right\}_{\Theta}, e_{2}\right\}_{\Theta}, \forall e_{1}, e_{2} \in \Gamma(E)
$$

Let us consider the general case where $H \neq 0$. Then the canonical function $\alpha$ satisfies

$$
\frac{1}{2}\left\{\alpha_{\mathcal{L}}, \alpha_{\mathcal{L}}\right\}_{\Theta}=-\left.\frac{1}{4 !}\{\{\{\{H, \alpha\}, \alpha\}, \alpha\}, \alpha\}\right|_{T^{*}[2] E[1]} \neq 0,
$$


from the canonical function equation. This is a twisted QP manifold $T^{*}[2] E[1]$ of degree 2. The structure given by Eqs. (4.4), (4.5), and (4.6) is the $H_{4}$-twisted Courant algebroid, ${ }^{20}$ in which the Leibniz identity of the Dorfman bracket $(4.7)$ on $\Gamma(E)$ is broken by the 4-form $H$ :

$$
\left[e_{1},\left[e_{2}, e_{3}\right]_{D}\right]_{D}-\left[\left[e_{1}, e_{2}\right]_{D}, e_{3}\right]_{D}-\left[e_{2},\left[e_{1}, e_{3}\right]_{D}\right]_{D}=\wedge^{4} \rho^{\#} H .
$$

Note that on $M, H$ is a 4-form and $h$ is a 3-form. Such a 4-form twisted Courant algebroid appears naturally in the theory of three-dimensional topological sigma models with Wess-Zumino terms, the cotangent extension of quadratic transitive Lie algebroids, and the reduction of exact Courant algebroids. It also naturally arises from a coisotropic Cartan geometry; see Ref. 49.

Example 4.3 (Twisted higher Dorfman brackets of degree $n$ ). Let $E \longrightarrow M$ be a vector bundle over a manifold $M$. We take an N-manifold $\left(T^{*}[n] T^{*}[n-1] E[1], \omega, \Theta\right)$ with a canonical function $\alpha$. Local coordinates on $\mathcal{L}=T^{*}[n-1] E[1]$ are chosen as $\left(x^{i}, u^{a}, p_{i}, w_{a}\right)$ of degree $(0,1$, $n-1, n-2)$, and those of the fiber are chosen as $\left(\xi_{i}, v_{a}, q^{i}, z^{a}\right)$ of degree $(n, n-1,1,2)$.

The canonical graded symplectic structure of degree $n$ is given by

$$
\omega=\delta x^{i} \wedge \delta \xi_{i}+(-1)^{n} \delta u^{a} \wedge \delta v_{a}+\delta p_{i} \wedge \delta q^{i}+(-1)^{n} \delta w_{a} \wedge \delta z^{a} .
$$

We define a Lagrangian submanifold by $\mathcal{L}=T^{*}[n-1] E[1]=\left\{\xi_{i}=v_{a}=q^{i}=z^{a}=0\right\}$ and a Q-structure satisfying $\left.\Theta\right|_{\mathcal{L}}=0$ as

$$
\Theta:=\xi_{i} q^{i}+v_{a} z^{a}+\frac{1}{2} C^{a}{ }_{i j}(x) v_{a} q^{i} q^{j}+\frac{1}{(n+1) !} H_{i_{0} \cdots i_{n}}(x) q^{i_{0}} \cdots q^{i_{n}} .
$$

Then $\{\Theta, \Theta\}=0$ produces $d H=0$ and $d C=0$, where $H=\frac{1}{(n+1) !} H_{i_{0} \cdots i_{n}}(x) d x^{i_{0}} \wedge \cdots \wedge d x^{i_{n}}$ is a $(n+1)$-form, and $C^{a}=\frac{1}{2} C^{a}{ }_{i j}(x) d x^{i} \wedge d x^{j}$ is a 2 -form taking values in $E^{*}$

We take the following canonical function $\alpha$ :

$$
\alpha=\rho_{a}^{i}(x) u^{a} p_{i}+\frac{1}{2} f^{a}{ }_{b c}(x) w_{a} u^{b} u^{c}+\frac{1}{n !} h_{a_{1} \cdots a_{n}}(x) u^{a_{1}} \cdots u^{a_{n}} .
$$

Direct calculation shows that $\left.e_{\alpha}^{\delta} \Theta\right|_{\mathcal{L}}=0$ produces the equations:

$$
\begin{aligned}
& \frac{\partial \rho^{i}{ }_{a}}{\partial x^{j}} \rho_{b}^{j}-\frac{\partial \rho^{i}{ }_{b}}{\partial x^{j}} \rho^{j}{ }_{a}+\rho^{i}{ }_{c} f^{c}{ }_{a b}-C^{a}{ }_{k l} \rho_{c}^{i}{ }_{c} \rho^{k}{ }_{a} \rho_{b}{ }_{b}=0, \\
& \frac{\partial f^{a}{ }_{b c}}{\partial x^{i}} \rho^{i}{ }_{d}+f^{a}{ }_{b e} f^{e}{ }_{c d}-C^{e}{ }_{i j} f_{b e}^{a} \rho^{i}{ }_{c} \rho^{j}{ }_{d}+(b c d \text { completely skewsymmetric })=0, \\
& \frac{\partial h_{a_{0} \cdots a_{n-1}}}{\partial x^{i}} \rho_{a_{n}}^{i}+h_{e a_{2} \cdots a_{n}} f_{a_{0} a_{1}}^{e}+C^{e}{ }_{i j} h_{e a_{0} \cdots a_{n-2}} \rho_{a_{n-1}} \rho^{j}{ }_{a_{n}}+H_{j_{0} \cdots j_{n}}(x) \rho^{j_{1}} a_{0} \cdots \rho^{j_{n}} a_{n} \\
& +\left(a_{0} \cdots a_{n} \text { completely skewsymmetric }\right)=0 .
\end{aligned}
$$

Note that the derived bracket $\{-,-\}_{\Theta}$ is just the canonical nondegenerate Poisson bracket on $\mathcal{L}=T^{*}[n-1] E[1]$. Since $\Gamma\left(E \otimes \wedge^{n-2} E^{*}\right)$ is identified as a subspace of $C^{\infty}\left(T^{*}[n] E[1]\right)$, we can define a bracket on $\Gamma\left(E \otimes \wedge^{n-2} E^{*}\right)$ by the derived-derived bracket:

$$
[-,-]_{D}=-\left\{\left\{-, \alpha_{\mathcal{L}}\right\}_{\Theta},-\right\}_{\Theta} .
$$

If $C=H=0$, we have $\left\{\alpha_{\mathcal{L}}, \alpha_{\mathcal{L}}\right\}_{\Theta}=0$. From Eqs. (4.10), (4.11), and (4.12), [ --$]_{D}$ is just the higher Dorfman bracket on $E \otimes \wedge^{n-2} E^{*}$. If $C$ or $H$ is nonzero, $\left(T^{*}[n-1] E[1],\{-,-\}_{\Theta}, \alpha_{\mathcal{L}}\right)$ is a twisted QP manifold. In particular, if $C=0$ and $H$ is nonzero, we obtain a twisted higher Dorfman bracket where the Leibniz identity of the Dorfman bracket is broken by a closed $n+1$-form $H$. 
Example 4.4. A Nambu-Poisson bracket of order $n(\geq 3)$ on $M$ is a skewsymmetric linear map $\{\cdot, \cdots, \cdot\}: C^{\infty}(M)^{\otimes n} \longrightarrow C^{\infty}(M)$ such that

(1) $\left\{f_{\sigma(1)}, f_{\sigma(2)}, \cdots, f_{\sigma(n)}\right\}=(-1)^{\epsilon(\sigma)}\left\{f_{1}, f_{2}, \cdots, f_{n}\right\}$,

(2) $\left\{f_{1} g_{1}, f_{2}, \cdots, f_{n}\right\}=f_{1}\left\{g_{1}, f_{2}, \cdots, f_{n}\right\}+g_{1}\left\{f_{1}, f_{2}, \cdots, f_{n}\right\}$,

(3) $\left\{f_{1}, f_{2}, \cdots, f_{n-1},\left\{g_{1}, g_{2}, \cdots, g_{n}\right\}\right\}$

$=\sum_{k=1}^{n}\left\{g_{1}, \cdots, g_{k},\left\{f_{1}, f_{2}, \cdots, f_{n-1}, g_{k}\right\}, g_{k+1}, \cdots, g_{n}\right\}$.

The $n$-vector field $\pi \in \Gamma\left(\wedge^{n} T M\right)$ is called the Nambu-Poisson tensor field, and it is defined as

$$
\pi\left(d f_{1}, d f_{2}, \cdots, d f_{n}\right)=\left\{f_{1}, f_{2}, \cdots, f_{n}\right\} .
$$

Let us assume that the Nambu-Poisson tensor is decomposable. Then a canonical function for the Nambu-Poisson structure is constructed as a special case of Example 4.3, as follows. Consider an N-manifold $T^{*}[n] \mathcal{L}=T^{*}[n] T^{*}[n-1] E[1]$, where $E=\wedge^{n-1} T^{*} M$. We take local coordinates $\left(x^{i}, v_{I}, p_{i}, w^{I}\right)$ of degree $(0,1, n-1, n-2)$ on $T^{*}[n-1] E[1]$ and conjugate local coordinates $\left(\xi_{i}, u^{I}, q^{i}, z_{I}\right)$ of degree $(n, n-1,1,2)$ on the fiber, where $I$ is the multi-index $I=\left(i_{1}, i_{2}, \cdots, i_{n}-1\right)$.

The canonical graded symplectic structure of degree $n$ can be expressed as

$$
\omega=\delta x^{i} \wedge \delta \xi_{i}+(-1)^{n} \delta v_{I} \wedge \delta u^{I}+\delta p_{i} \wedge \delta q^{i}+(-1)^{n} \delta w^{I} \wedge \delta z_{I}
$$

A Q-structure function $\Theta$ is defined as

$$
\Theta=-q^{i} \xi_{i}+\frac{1}{(n-1) !} z_{I}\left(u^{I}-q^{i_{1}} \cdots q^{i_{n-1}}\right),
$$

which automatically satisfies $\{\Theta, \Theta\}=0$. $\Theta$ defines the Dorfman bracket on $T M \oplus \wedge^{n-1} T^{*} M^{13}$ by the derived bracket $[-,-]_{D}=-\{\{\Theta,-\},-\}$. We take the function $\alpha$ to be

$$
\alpha=-\frac{1}{(n-1) !} \pi^{i_{1} \cdots i_{n-1} i_{n}}(x) v_{i_{1} \cdots i_{n-1}} p_{i_{n}} .
$$

Proposition 4.3. Let $\mathcal{L}=T^{*}[n-1] E[1]$ be the Lagrangian submanifold of $T^{*}[n] \mathcal{L}$. Then $\alpha$ is a canonical function with respect to $\Theta$ and $\mathcal{L}$, i.e., $\left.e^{\delta_{\alpha}} \Theta\right|_{\mathcal{L}}=0$ if and only if $\pi$ is a decomposable Nambu-Poisson tensor. ${ }^{4}$

\section{Strong Courant algebroids}

Let $E$ and $A$ be two vector bundles on $M$. We consider an N-manifold $T^{*}[3] \mathcal{L}=T^{*}[3](E[1] \oplus$ $A[2])$. Let us take local coordinates $\left(x^{i}, u^{a}, z_{p}\right)$ on $M, E[1]$, and $A[2]$, and local coordinates $\left(\xi_{i}, v_{a}, w^{p}\right)$ of the fiber of $T^{*}[3]$ of degree $(3,2,1)$, respectively.

A canonical graded symplectic structure is $\omega=\delta x^{i} \wedge \delta \xi_{i}-\delta u^{a} \wedge \delta v_{a}+\delta w^{p} \wedge \delta z_{p}$. We define a Q-structure function as

$$
\Theta=\frac{1}{2} k^{a b} v_{a} v_{b}+\rho_{r}^{i}(x) \xi_{i} w^{r}+\frac{1}{2} C_{p q}^{r}(x) z_{r} w^{p} w^{q},
$$

where $k^{a b}$ is a fiber metric on $E .\{\Theta, \Theta\}=0$ is equivalent to the following identities:

$$
\begin{aligned}
& \rho^{i}{ }_{r} \frac{\partial \rho^{i}{ }_{s}}{\partial x^{j}}-\rho^{i}{ }_{s} \frac{\partial \rho^{i}{ }_{r}}{\partial x^{j}}-\rho_{p}^{i} C_{r s}^{p}=0, \\
& -\rho^{i}{ }_{p} \frac{\partial C^{s}{ }_{q r}}{\partial x^{i}}+C^{s}{ }_{p t} C^{t q r}+(p q r \text { cyclic })=0 .
\end{aligned}
$$

This condition is satisfied if $(A, C, \rho)$ is a Lie algebroid, where a Lie bracket is $\left[e_{p}, e_{q}\right]_{A}=C^{r p q}(x) e_{r}$ and $\rho$ is a bundle map from $A$ to $T M$ defined by $\rho\left(e_{r}\right)=\rho^{i}{ }_{r}(x) \frac{\partial}{\partial x^{i}}$ for $e_{r} \in \Gamma A$. 
Let the Lagrangian submanifold $\mathcal{L} \subset T^{*}[3] \mathcal{L}$ be $\left\{\xi_{i}=v_{a}=w^{p}=0\right\}$, and let a canonical function of degree 3 with respect to the Lagrangian submanifold $\mathcal{L}$ be

$$
\alpha=\tau^{r}{ }_{a}(x) z_{r} u^{a}+\frac{1}{3 !} h_{a b c}(x) u^{a} u^{b} u^{c} .
$$

The canonical function equation $\left.e^{\delta_{\alpha}} \Theta\right|_{\mathcal{L}}=0$ is equivalent to $\left.\Theta\right|_{\mathcal{L}^{\prime}}=0$, where $\mathcal{L}^{\prime}$ is a Lagrangian submanifold with respect to the inverse canonical transformation of the P-structure, $\omega^{\prime}=-d\left(e^{-\delta_{\alpha}} \vartheta\right)$. $\mathcal{L}^{\prime}$ is defined by

$$
\begin{aligned}
\xi_{i} & =\left\{\xi_{i}, \alpha\right\}=-\frac{\partial \tau^{r}{ }_{a}}{\partial x^{i}}(x) z_{r} u^{a}-\frac{1}{3 !} \frac{\partial h_{a b c}}{\partial x^{i}}(x) u^{a} u^{b} u^{c}, \\
v_{a} & =\left\{v_{a}, \alpha\right\}=-\tau^{r}{ }_{a}(x) z_{r}-\frac{1}{2} h_{a b c}(x) u^{b} u^{c}, \\
w^{r} & =\left\{w^{r}, \alpha\right\}=\tau^{r}{ }_{a}(x) u^{a} .
\end{aligned}
$$

Substituting this equation into $\left.\Theta\right|_{\mathcal{L}^{\prime}}=0$, we obtain the conditions for the canonical function, as follows:

$$
\begin{aligned}
& k^{a b} \tau^{r}{ }_{a} \tau_{b}^{s}=0, \\
& k^{c d} \tau^{r}{ }_{c} h_{d a b}+\rho_{s}^{i} \tau^{s}{ }_{a} \frac{\partial \tau^{r}{ }_{b}}{\partial x^{i}}-\rho_{s}^{i} \tau^{s}{ }_{b} \frac{\partial \tau^{r}{ }_{a}}{\partial x^{i}}+C^{r}{ }_{p q} \tau^{p}{ }_{a} \tau^{q}{ }_{b}=0, \\
& \rho^{i}{ }_{r} \tau^{r}{ }_{d} \frac{\partial h_{a b c}}{\partial x^{i}}-\frac{1}{2} k^{e f} h_{e a b} h_{f c d}+(a b c d \text { complete skewsymmetric })=0 .
\end{aligned}
$$

Now let us analyze the geometric structure defined by this canonical function. Let $\tau: E \longrightarrow A$ be the bundle map defined by the above $\tau_{d}^{r}$ on local charts. Moreover, we define the following operations by the derived-derived bracket:

$$
\begin{aligned}
{\left[e_{1}, e_{2}\right]_{D} } & =-\left\{\left\{e_{1}, \alpha_{\mathcal{L}}\right\}_{\Theta}, e_{2}\right\}_{\Theta}, \\
\left\langle e_{1}, e_{2}\right\rangle & =\left\{e_{1}, e_{2}\right\}_{\Theta}, \\
\mathcal{D}(f) & =\left\{\alpha_{\mathcal{L}}, f\right\}_{\Theta},
\end{aligned}
$$

where $\{-,-\}_{\Theta}:=-\left.\{\{-, \Theta\},-\}\right|_{\mathcal{L}}$ is the derived bracket, $[-,-]_{D}$ is a bilinear bracket, $\langle-,-\rangle$ is an inner product on $\Gamma(E)$, and $\mathcal{D}$ is a map from $C^{\infty}(M)$ to $\Gamma(E)$.

By Eqs. (4.18), (4.19), and (4.20) for $e_{1}, e_{2}, e_{3} \in \Gamma(E)$ and $\xi_{1}, \xi_{2} \in \Gamma\left(A^{*}\right)$, we have

(a) $\tau\left[e_{1}, e_{2}\right]_{D}=\left[\tau e_{1}, \tau e_{2}\right]_{A}$,

(b) $\left\langle\tau *\left(\xi_{1}\right), \tau *\left(\xi_{2}\right)\right\rangle=0$,

(c) $\left[e_{1}, e_{1}\right]_{D}=\mathcal{D}\left\langle e_{1}, e_{1}\right\rangle=(\rho \circ \tau)^{*} d\left\langle e_{1}, e_{1}\right\rangle$,

(d) $\left[e_{1},\left[e_{2}, e_{3}\right]_{D}\right]_{D}=\left[\left[e_{1}, e_{2}\right]_{D}, e_{3}\right]_{D}+\left[e_{2},\left[e_{1}, e_{3}\right]_{D}\right]_{D}$,

(e) $\rho \circ \tau\left(e_{1}\right)\left\langle e_{2}, e_{3}\right\rangle=\left\langle\left[e_{1}, e_{2}\right]_{D}, e_{3}\right\rangle+\left\langle e_{2},\left[e_{1}, e_{3}\right]_{D}\right\rangle$.

We call this structure a strong Courant algebroid.

Definition 4.4. A strong Courant algebroid is $\left(E, \tau,\langle-,-\rangle,[-,-]_{D}, A, \rho,[-,-]_{A}\right)$ satisfying the equation $(a)-(e)$, where $E$ is a vector bundle on a manifold $M,\langle-,-\rangle$ is an inner product on $E,[-,-]_{D}$ is a bilinear operator, $\left(A, \rho,[-,-]_{A}\right)$ is a Lie algebroid on $M$, and $\tau: E \longrightarrow A$ is a bundle map.

Roughly speaking, a strong Courant algebroid is a Courant algebroid over a Lie algebroid. Obviously, any Courant algebroid is naturally a strong Courant algebroid.

Proposition 4.5. Let $\left(E, \tau,\langle-,-\rangle,[-,-]_{D}, A, \rho,[-,-]_{A}\right)$ be a strong Courant algebroid. Then $\left(E, \tau \circ \rho,\langle-,-\rangle,[-,-]_{D}\right)$ is a Courant algebroid. 
Thus for any strong Courant algebroid, there exists a Courant algebroid associated with it.

Example 4.5. If $M$ is a point, the strong Courant algebroid is a triple $\left(\mathfrak{g}_{1}, \mathfrak{g}_{2}, \tau\right)$, where $\mathfrak{g}_{1}$ is a quadratic Lie algebra, $\mathfrak{g}_{2}$ is a Lie algebra, and $\tau$ is a homomorphism from $\mathfrak{g}_{1}$ to $\mathfrak{g}_{2}$ such that $\tau^{*}\left(\mathfrak{g}_{2}^{*}\right)$ is an isotropic subspace of $\mathfrak{g}_{1}$. Any Lie bialgebra is an example of the strong Courant algebroid.

Example 4.6. Let $A$ be a Lie algebroid. An inner product on $A \oplus A^{*}$ is defined by the natural pairing of $A$ and $A^{*}$, an anchor map $\tau$ is defined by the natural projection from $A \oplus A^{*}$ to $A$. It is clear that $\left(A \oplus A^{*}, \tau, A,[-,-]_{D}\right)$ gives a strong Courant algebroid, where $[-,-]_{D}$ is the Dorfman bracket given by

$$
[X+\xi, Y+\eta]_{D}=[X, Y]_{A}+L_{X} \eta-i_{Y} d \xi
$$

$X, Y \in \Gamma(A), \xi, \eta \in \Gamma\left(A^{*}\right)$, and $L$ and $d$ are the Lie derivative and the de Rham differential associated to $A$, respectively.

Example 4.7. Let $P$ be a $G$-principal bundle over $M$. Since $G$ acts on $T P \oplus T^{*} P$ naturally, we get a bundle $\frac{T P \oplus T^{*} P}{G}$ over $M$ by reduction, where the $G$-invariant sections of $T P \oplus T^{*} P$ reduce to the sections of the bundle $\frac{T P \oplus T^{*} P}{G}$.

We define a bundle map $\tau$ by the natural projection from $\frac{T P \oplus T^{*} P}{G}$ to the Atiyah algebroid $\frac{T P}{G}$. Because of the $G$-invariance, the canonical Dorfman bracket and the natural pairing on $T P \oplus T^{*} P$ induce a bracket $[-,-]_{D}$ and an inner product $\langle-,-\rangle$ on $\Gamma\left(\frac{T P \oplus T^{*} P}{G}\right)$. It is easy to confirm that $\left(\frac{T P \oplus T^{*} P}{G}, \tau,\langle-,-\rangle,[-,-]_{D}, \frac{T P}{G}\right)$ is a strong Courant algebroid.

Following the discussion in Subsection IV A, we can easily generalize the strong Courant algebroid to the twisted version. For most of the concepts that appear in the Courant algebroids, parallels can be introduced in the strong Courant algebroids. The A-connections and morphisms between Lie algebroids ${ }^{14}$ will play a key role in the further study of the strong Courant algebroids.

\section{TWISTED QP MANIFOLDS AND BULK-BOUNDARY AKSZ SIGMA MODELS}

\section{A. QP manifolds as QP pairs}

As a physical application, we will show how a QP pair encodes the bulk-boundary correspondence of AKSZ sigma models.

The following theorem justifies the view that a twisted QP manifold is a generalization of a QP manifold.

Theorem 5.1. A QP manifold is a twisted $Q P$ manifold.

Proof. Let $\left(\mathcal{L}, \omega_{\mathcal{L}}, \alpha_{\mathcal{L}}\right)$ be a QP manifold of degree $n$. We consider the Poisson bracket $\{-,-\}_{\mathcal{L}}$ for the symplectic structure $\omega_{\mathcal{L}}$. We can construct a Poisson bivector field $\pi_{\mathcal{L}}$ to define the Poisson bracket $\{f, g\}_{\mathcal{L}}=\pi_{\mathcal{L}}(\delta f, \delta g)$, where $f, g \in C^{\infty}(\mathcal{L})$ and $\delta$ is a differential on $\mathcal{L}$.

Consider a shifted cotangent bundle $T^{*}[n+1] \mathcal{L}$, and choose a canonical graded symplectic structure $\omega=\omega_{\text {can }}$ on $T^{*}[n+1] \mathcal{L}$. From the theory of supergeometry, we have that a bivector field on $\mathcal{L}$ can be identified as a function on $T^{*}[n+1] \mathcal{L}$. Therefore we obtain $\Theta \in C^{\infty}\left(T^{*}[n+1] \mathcal{L}\right)$ corresponding to $\pi_{\mathcal{L}}$. We can easily prove that $\pi_{\mathcal{L}}(\delta f, \delta g)=\left\{\left\{\pi^{*} f, \Theta\right\}_{\text {can }}, \pi^{*} g\right\}_{\text {can }} \mid \mathcal{L}$, which is similar to the formula for the usual Poisson bracket. Therefore the small Poisson bracket $\{-,-\}_{\mathcal{L}}$ is rederived from the derived bracket of the big bracket.

Note that $\alpha=\pi^{*} \alpha_{\mathcal{L}}$ is a canonical function for this $\Theta$ because $\left\{\alpha_{\mathcal{L}}, \alpha_{\mathcal{L}}\right\}_{\mathcal{L}}=$ $\left.\left\{\{\alpha, \Theta\}_{\text {can }}, \alpha\right\}_{\text {can }}\right|_{\mathcal{L}}=0$. Therefore, $\left(T^{*}[n+1] \mathcal{L}, \omega_{\text {can }}, \Theta, \alpha\right)$ is a QP pair, and the original QP manifold $\mathcal{L}$ is its small QP manifold.

Let us describe it using a local coordinate. Let $q^{i}$ be a local coordinate of degree $\left|q^{i}\right|$ on $\mathcal{L}$ such that $\left\{q^{i}, q^{j}\right\}_{\mathcal{L}}=\left(\omega_{\mathcal{L}}^{-1}\right)^{i j}(q)$. There exists a local Darboux coordinate $p_{i}$ of degree $\left|p_{i}\right|$ on the fiber of $T^{*}[n+1] \mathcal{L}$ such that

$$
\left\{q^{i}, p_{j}\right\}_{c a n}=-(-1)^{\left(\left|q^{i}\right|-n-1\right)\left(\left|p_{j}\right|-n-1\right)}\left\{p_{j}, q^{i}\right\}_{c a n}=\delta^{i}{ }_{j}
$$


where $\{-,-\}_{c a n}$ is the Poisson bracket defined from the canonical graded symplectic form $\omega_{\text {can }}$ on $T^{*}[n+1] \mathcal{L}$. If we define $\Theta=-(-1)^{\left(\left|q^{i}\right|-n-1\right)\left(\left|p_{j}\right|-n-1\right)} \frac{1}{2}\left(\omega_{\mathcal{L}}^{-1}\right)^{i j}(q) p_{i} p_{j}$, then a straightforward computation gives $\{\Theta, \Theta\}_{c a n}=0$ and $\{-,-\}_{\mathcal{L}}=\left.\left\{\{-, \Theta\}_{c a n},-\right\}_{c a n}\right|_{\mathcal{L}}$.

This construction shows that all the terms $\left.\Theta\right|_{\mathcal{L}},\left.\{\Theta, \alpha\}_{c a n}\right|_{\mathcal{L}},\left.\left\{\{\Theta, \alpha\}_{c a n}, \alpha\right\}_{c a n}\right|_{\mathcal{L}}$,

$\left\{\left\{\{\Theta, \alpha\}_{c a n}, \alpha\right\}_{c a n}, \alpha\right\}_{c a n} \mid \mathcal{L}, \cdots$, are zero for the canonical $\Theta$. Therefore, we obtain

$$
\begin{aligned}
\left.e^{\delta_{\alpha}} \Theta\right|_{\mathcal{L}} & =\left.\Theta\right|_{\mathcal{L}}+\left.\{\Theta, \alpha\}_{c a n}\right|_{\mathcal{L}}+\left.\frac{1}{2}\left\{\{\Theta, \alpha\}_{c a n}, \alpha\right\}_{c a n}\right|_{\mathcal{L}}+\left.\frac{1}{3 !}\left\{\left\{\{\Theta, \alpha\}_{c a n}, \alpha\right\}_{c a n}, \alpha\right\}_{c a n}\right|_{\mathcal{L}}+\cdots \\
& =0 .
\end{aligned}
$$

Thus, $\alpha$ is a canonical function.

From the proof of this theorem, there exists a QP pair for any QP manifold.

\section{B. Twisted QP manifolds from deformations}

In this subsection, we show how twisted QP structures on $\mathcal{L}$ can be obtained by the deformation of the canonical $\Theta$ on $T^{*}[n+1] \mathcal{L}$. As an application, we can add "fluxes" to $\mathcal{L}$ by deforming the corresponding canonical homological function on $T^{*}[n+1] \mathcal{L}$. This also leads to a common method for constructing a twisted QP manifold.

First, we take a P-manifold $\left(\mathcal{L}, \omega_{\mathcal{L}}\right)$ of degree $n$. From the proof of Theorem 5.1, there exists a QP realization $\left(T^{*}[n+1] \mathcal{L},\{-,-\}, \Theta\right)$ with $\alpha=0$, where $\Theta$ is the canonical Q-structure. By definition, $\{-,-\}_{\Theta}=\left.\{\{-, \Theta\},-\}\right|_{\mathcal{L}}$ coincides with the original Poisson bracket derived from $\omega_{\mathcal{L}}$.

Next, we consider a deformation of $\Theta, \Theta_{d}=\Theta+\Theta^{\prime}$, such that $\left\{\Theta_{d}, \Theta_{d}\right\}=0$. This can be rewritten as the Maurer-Cartan equation, $\left\{\Theta, \Theta^{\prime}\right\}+\frac{1}{2}\left\{\Theta^{\prime}, \Theta^{\prime}\right\}=0$. For simplicity, we concentrate on the deformation in which the derived P-structure on $\mathcal{L}$ is not changed, i.e., $\left.\left\{\left\{-, \Theta_{d}\right\},-\right\}\right|_{\mathcal{L}}=$ $\left.\{\{-, \Theta\},-\}\right|_{\mathcal{L}}$. One such solution is the deformation for which $\left.\Theta^{\prime}\right|_{\mathcal{L}^{\perp}}=0$.

A function $\alpha$ of degree $n+1$ on $\mathcal{L}$ is a canonical function on $\left(T^{*}[n+1] \mathcal{L},\{-,-\}, \Theta_{d}\right)$ with respect to $\mathcal{L}$ if and only if

$$
\left.e^{\delta_{\alpha}} \Theta_{d}\right|_{\mathcal{L}}=\left.e^{\delta_{\alpha}}\left(\Theta+\Theta^{\prime}\right)\right|_{\mathcal{L}}=0 .
$$

From the above assumption, the equation $\left.e^{\delta_{\alpha}} \Theta\right|_{\mathcal{L}}=0$ is equivalent to the master equation $\left\{\alpha_{\mathcal{L}}, \alpha_{\mathcal{L}}\right\}_{\Theta}=0$ for the canonical homological function $\Theta$ on $T^{*}[n+1] \mathcal{L}$. However, Eq. (5.2) for $\Theta_{d}$ generally breaks the classical master equation. To see this, we recall that $\left.e^{\delta_{\alpha}} \Theta_{d}\right|_{\mathcal{L}}=0$ produces the equation

$$
\frac{1}{2}\left\{\alpha_{\mathcal{L}}, \alpha_{\mathcal{L}}\right\}_{\Theta_{d}}=-\left.\left(\Theta+\Theta^{\prime}\right)\right|_{\mathcal{L}}-\left.\left\{\Theta+\Theta^{\prime}, \alpha\right\}\right|_{\mathcal{L}}-\left.\frac{1}{3 !}\left\{\left\{\left\{\Theta+\Theta^{\prime}, \alpha\right\}, \alpha\right\}, \alpha\right\}\right|_{\mathcal{L}} \cdots .
$$

Note that for a canonical $\Theta$, the terms $\left.\Theta\right|_{\mathcal{L}}$ and $\left.\{\{\ldots\{\Theta, \alpha\}, \ldots, \alpha\}, \alpha\}\right|_{\mathcal{L}}$ on the right-hand side are equal to zero. However, the terms involving a higher bracket between $\Theta^{\prime}$ and $\alpha$, for example, $\left.\left\{\left\{\left\{\Theta^{\prime}, \alpha\right\}, \alpha\right\}, \alpha\right\}\right|_{\mathcal{L}}$, may be nonzero. Also, $\left\{\alpha_{\mathcal{L}}, \alpha_{\mathcal{L}}\right\}_{\Theta_{d}}$ is generally nonzero. Therefore, a solution $\alpha$ of the canonical function of Eq. (5.2) with respect to $\Theta_{d}$ gives a twisted QP manifold $\left(\mathcal{L},\{-,-\}_{\Theta_{d}}, \alpha_{\mathcal{L}}\right)$. The following general proposition is obtained.

Proposition 5.2. Let $\left(\mathcal{L}, \omega_{\mathcal{L}}\right)$ be a P-manifold, and let $\left(T^{*}[n+1] \mathcal{L}, \omega, \Theta\right)$ be the canonical $Q P$ realization given in Theorem 5.1. Then there is a twisted $Q P$ manifold $\left(\mathcal{L}, \omega_{\mathcal{L}}, \alpha_{\mathcal{L}}\right)$ associated with any function $\Theta^{\prime}$ of degree $n+2$ on $T^{*}[n+1] \mathcal{L}$ satisfying

1. $\left\{\Theta, \Theta^{\prime}\right\}+\frac{1}{2}\left\{\Theta^{\prime}, \Theta^{\prime}\right\}=0$,

2. $\left.\left\{\left\{-, \Theta^{\prime}\right\},-\right\}\right|_{\mathcal{L}}=0$,

3. $\left.e^{\delta_{\alpha}} \Theta^{\prime}\right|_{\mathcal{L}}=0$,

where $\alpha=\pi^{*} \alpha_{\mathcal{L}}$. In this case, we say that the twisted $Q P$ manifold $\left(\mathcal{L}, \omega, \alpha_{\mathcal{L}}\right)$ is twisted by $\Theta^{\prime}$.

One can find an equivalent description of a twisted QP manifold in the frame of homotopy Poisson manifolds in Ref. 34, where the authors encode deformations of the canonical homological 
function on $\left(T^{*}[n+1] \mathcal{L}, \omega\right)$ by higher derived brackets. A deformation of the homological function on $T^{*}[n+1] \mathcal{L}$ induces a homotopy Poisson algebra on $C^{\infty}(\mathcal{L})$, and the canonical transformation equation $\left.e^{\delta_{\alpha}} \Theta\right|_{\mathcal{L}}=0$ becomes the Maurer-Cartan equation for the underlying $L_{\infty}$-algebra, which can be seen as a homotopy version of the normal master equation. As a result, a twisted QP manifold can be viewed as a homotopy version of a QP manifold.

\section{Bulk-boundary correspondence of AKSZ sigma models}

Generally, a kind of (topological) quantum field theory in $n+1$ dimensions on $X$ has the same physical constants as a quantum field theory in $n$ dimensions on the boundary $\partial X$. Theorem 5.1 says that there is another QP manifold associated with any (twisted) QP manifold. Therefore, we obtain a pair of AKSZ sigma models associated with one QP manifold. This can be interpreted physically as the bulk-boundary correspondence (holographic correspondence) of physical models.

Let us take a QP pair $\left(T^{*}[n+1] \mathcal{L}, \omega, \Theta, \mathcal{L}, \alpha\right)$. First, we suppose that $\alpha$ satisfies $\left\{\alpha_{\mathcal{L}}, \alpha_{\mathcal{L}}\right\}_{\Theta}=$ $\left.\{\{\alpha, \Theta\}, \alpha\}\right|_{\mathcal{L}}=0$, i.e., $\left(\mathcal{L},\{-,-\}_{\Theta}, \alpha_{\mathcal{L}}\right)$ is a nontwisted QP manifold, where $\alpha=\pi^{*} \alpha_{\mathcal{L}}$ with $\pi$ : $T^{*}[n+1] \mathcal{L} \longrightarrow \mathcal{L}$.

We obtain two AKSZ sigma models from these data. Let $X$ be a manifold in $n+1$ dimensions with boundaries. By the AKSZ construction, the original big QP manifold $\left(T^{*}[n+1] \mathcal{L}, \omega, \Theta, \mathcal{L}, \alpha\right)$ defines an AKSZ sigma model with a boundary on $\mathcal{X}=T[1] X$. A P-structure is given by $\omega=$ $\mu_{*} \mathrm{ev}^{*} \omega$, and a Q-structure by (3.5) or, equivalently, by (3.6).

On the other hand, with the AKSZ construction, the small QP manifold $\left(\mathcal{L},\{-,-\}_{\Theta}, \alpha_{\mathcal{L}}\right)$ defines an AKSZ sigma model on $\partial \mathcal{X}=T[1] \partial X$. The Q-structure is

$$
\begin{aligned}
S_{\mathcal{L}} & =S_{\mathcal{L} 0}+S_{\mathcal{L} 1} \\
& =\iota_{\hat{D}_{\partial \mathcal{X}}} \mu_{\partial \mathcal{X}_{*}} \operatorname{ev}^{*} \vartheta_{\Theta}+\mu_{\partial \mathcal{X} *}\left(i_{\partial} \times \mathrm{id}\right)^{*} \operatorname{ev}^{*} \alpha,
\end{aligned}
$$

where $\omega_{\Theta}=-\delta \vartheta_{\Theta}$.

These two AKSZ sigma models, which are constructed from one QP pair, have a canonical correspondence. Physical arguments show that the BV actions $S$ and $S_{\mathcal{L}}$ correspond. First we consider the bulk Q-structure function (3.5) on $\mathcal{X}$. The Q-structure (5.3) on the Lagrangian submanifold $\mathcal{L}$ is obtained by using Stokes' theorem and integrating out the auxiliary superfields. (i.e., $S$ and $S_{\mathcal{L}}$ derive the same orbits of the equations of motion on $\partial \mathcal{X}$.) The boundary P-structure is obtained as $\omega_{\Theta}=-\delta \vartheta_{\Theta}$ from the first term $\iota_{\hat{D}_{\partial X}} \mu_{\partial \mathcal{X}{ }_{*}} \mathrm{ev}^{*} \vartheta_{\Theta}$ in the resulting Q-structure $S_{\mathcal{L}}$. This AKSZ sigma model on $\partial \mathcal{X}$ is equivalent to the one constructed from the small QP manifold.

The field configurations for the two AKSZ sigma models satisfy the following commutative diagram:

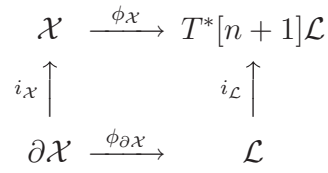

where $\phi_{\mathcal{X}} \in \operatorname{Map}\left(\mathcal{X}, T^{*}[n+1] \mathcal{L}\right), \phi_{\partial \mathcal{X}} \in \operatorname{Map}(\partial \mathcal{X}, \mathcal{L}), i_{\mathcal{X}}$ is the inclusion of the boundary into the manifold, and $i_{\mathcal{L}}$ is the inclusion as the zero section such that $\pi \circ i_{\mathcal{L}}=\mathrm{id}_{\mathcal{L}}$. This demonstrates the bulk-boundary correspondence of AKSZ sigma models.

We denote a QP pair $\left(T^{*}[n+1] \mathcal{L}, \omega, \Theta, \mathcal{L}, \alpha\right)$ by $\mathbf{Q P}_{T^{*}[n+1] \mathcal{L}}$ and a $\mathrm{QP}$ manifold without a canonical function $\left(\mathcal{L}, \omega_{s}, \alpha\right)$ by $\mathbf{Q P}_{\mathcal{L}}^{0}$. Let $\mathbf{A K S Z}_{\mathcal{X}, \mathcal{L}}$ be an $\operatorname{AKSZ}$ sigma model on $\operatorname{Map}(\mathcal{X}, \mathcal{L})$, and let $\mathbf{A K S Z}_{\mathcal{X}, \mathcal{L}}^{0}$ be an AKSZ sigma model without a boundary (without a canonical function). Schematically, the following diagram shows two procedures:

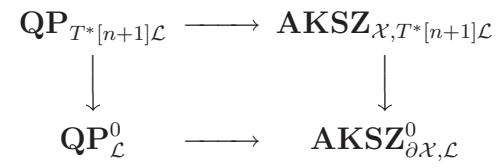


Thus, these two methods produce the same AKSZ sigma model $\mathbf{A K S Z}_{\partial \mathcal{X}, \mathcal{L}}^{0}$. In one method, we construct an AKSZ sigma model with a boundary and then reduce the theory to the boundary. In the other method, we reduce a QP pair to a small QP manifold and then construct an AKSZ sigma model.

Conversely, we can take a QP manifold of degree $n,\left(\mathcal{L},\{-,-\}_{\mathcal{L}}, \alpha_{\mathcal{L}}\right)$. Following Theorem 5.1, we have a canonical lift $\left(T^{*}[n+1] \mathcal{L}, \omega, \Theta, \mathcal{L}, \alpha\right)$. Although in general, a big QP manifold is not unique to one small QP manifold, we can show that for any lift $\left(T^{*}[n+1] \mathcal{L}, \omega, \Theta, \mathcal{L}, \alpha\right)$, the resulting small AKSZ sigma models on $\mathcal{L}$ have the equivalent QP structures. The equivalence relation for $\Theta$ for a fixed $\omega$ has the following physical interpretation in the AKSZ sigma models. Two Q-structure functions are equivalent, $S_{T^{*}[n+1] \mathcal{L}}^{\prime} \sim S_{T^{*}[n+1] \mathcal{L}}$, if $S_{T^{*}[n+1] \mathcal{L}}^{\prime}=S_{T^{*}[n+1] \mathcal{L}}+S_{\text {bulk }}$ on $\mathcal{X}$ have the same boundary conditions, that is, $S_{b u l k}$ has the boundary condition such that $\left.S_{b u l k}\right|_{\partial \mathcal{X}}=0$.

\section{Bulk-boundary correspondence of twisted AKSZ sigma models induced from general QP pairs}

In this subsection, we consider general cases, i.e., $\left\{\alpha_{\mathcal{L}}, \alpha_{\mathcal{L}}\right\}_{\Theta} \neq 0$. Given a QP pair, $\left(T^{*}[n+\right.$ $1] \mathcal{L}, \omega, \Theta, \mathcal{L}, \alpha)$, then by definition, $\left(\mathcal{L},\{-,-\}_{\mathcal{L}}, \alpha_{\mathcal{L}}\right)$ is a twisted QP manifold, where $\pi: T^{*}[n+$ $1] \mathcal{L} \longrightarrow \mathcal{L}$ is a natural projection and $\alpha=\pi^{*} \alpha_{\mathcal{L}}$.

Let us consider the AKSZ sigma models. Let $X$ be a manifold in $n+1$ dimensions with a boundary. By the AKSZ construction in Subsection II B, there is an AKSZ sigma model with a boundary from $\mathcal{X}=T$ [1] $X$ to $T^{*}[n+1] \mathcal{L}$. The Q-structure function is given in Eq. (3.5). A boundary theory can be constructed by the method inspired by physical arguments. The field configurations for two AKSZ sigma models are required to satisfy the following commutative diagram:

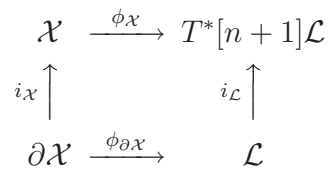

where $\pi \circ i_{\mathcal{L}}=\mathrm{id}_{\mathcal{L}}$. We restrict the equations of motion of the big AKSZ sigma model on $\mathcal{X}$ to the boundary $\partial \mathcal{X}$. The derived bracket $\mathrm{P}$-structure and the Q-structure function on the Lagrangian submanifold $\operatorname{Map}(\partial \mathcal{X}, \mathcal{L})$ are constructed by using Stokes' theorem and integrating out the auxiliary superfields from the bulk Q-function $S$, in a manner similar to that for the AKSZ sigma model cases in Subsection V C. ${ }^{38,4}$

The P-structure on $\operatorname{Map}(\partial \mathcal{X}, \mathcal{L})$ is the same as the $\mathrm{AKSZ}$ construction on a small P-manifold. The Poisson bracket derived from $\omega_{\mathcal{L}}=\mu_{\mathcal{L} *} \mathrm{ev}^{*} \omega_{\mathcal{L}}$ on $\mathcal{L}$ is equal to the derived bracket $\left.\{\{-,-\},-\}\right|_{\mathcal{L}}$ with respect to $\omega_{\mathcal{L}}$.

The bulk Q-structure is expressed by Eq. (3.5), and we use the equivalent Q-structure function (3.6). By restricting $\operatorname{Map}(\mathcal{X}, \mathcal{M})$ to orbits of solutions in the equations of motion with respect to the compliment space $\mathcal{L}^{\perp}=T^{*}[n+1] \mathcal{L} / \mathcal{L}$ by solving $\delta_{\mathcal{L}^{\perp}} S^{\prime \prime}=0$ and using Stokes' theorem, the bulk Q-structure $S^{\prime \prime}$ reduces to the boundary twisted Q-structure $S_{\mathcal{L}}$. We will demonstrate this procedure in Example 5.1. $S_{\mathcal{L}}$ has extra terms that are not generated by the original AKSZ construction on $\operatorname{Map}(T[1] \partial X, \mathcal{L})$. A direct calculation shows that the boundary Q-structure function is the following untwisted Q-structure function plus extra terms:

$$
\begin{aligned}
S_{\mathcal{L}}= & S_{\mathcal{L}, \partial \mathcal{X}}+S_{\mathcal{L}, \mathcal{X}} \\
= & \iota_{\hat{D}_{\partial X}} \mu_{\partial \mathcal{X} *} \mathrm{ev}^{*} \vartheta_{\Theta}-\mu_{\partial \mathcal{X} *}\left(i_{\partial} \times \mathrm{id}\right)^{*} \mathrm{ev}^{*} \alpha \\
& +\left.\left(\mu_{*} \mathrm{ev}^{*} \Theta-\frac{1}{2} \mu_{*} \mathrm{ev}^{*}\{\{\vartheta, \alpha\}, \alpha\} \cdots\right)\right|_{\delta_{\mathcal{L}^{\perp} S=0},}
\end{aligned}
$$

where $S_{\mathcal{L}, \partial \mathcal{X}}$ is a Q-structure function in the AKSZ construction, $S_{\mathcal{L}, \mathcal{X}}$ is an extra term from the integration over $\mathcal{X}$. (Note that $\mu_{*}$ is the integration over $\mathcal{X}$.) The last terms are physically called Wess-Zumino terms. In general, the master equation is not satisfied because of the presence of $S_{\mathcal{L}, \mathcal{X}}$. If $\{\alpha, \alpha\}=0$ but $\left\{\alpha_{\mathcal{L}}, \alpha_{\mathcal{L}}\right\}_{\Theta} \neq 0$, the WZ terms can be written as the following expression by using 
Eq. (3.7):

$$
S_{\mathcal{L}}=\iota_{\hat{D}_{\partial X}} \mu_{\partial \mathcal{X}_{*}} \mathrm{ev}^{*} \vartheta_{\Theta}-\mu_{\partial \mathcal{X}_{*}}\left(i_{\partial} \times \mathrm{id}\right)^{*} \mathrm{ev}^{*} \alpha+\left.\mu_{*} \mathrm{ev}^{*} \Theta\right|_{\delta_{\mathcal{L}^{\perp}} S=0} .
$$

We call this construction of a topological sigma model, $\left(\operatorname{Map}(\partial \mathcal{X}, \mathcal{L}),\{-,-\}_{\mathcal{L}}, S_{\mathcal{L}}\right)$, the twisted AKSZ construction, and we call the resulting model the twisted AKSZ sigma model. The following schematic diagram expresses our construction procedure:

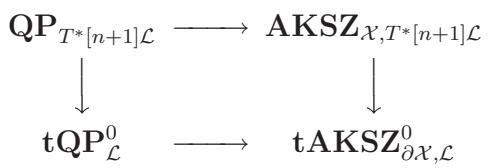

Here $\mathbf{t} \mathbf{Q P}{ }_{\mathcal{L}}^{0}$ is a twisted QP manifold on $\mathcal{L}$ and $\mathbf{t} \mathbf{A K S Z} \mathbf{Z}_{\partial \mathcal{X} \mathcal{L}}^{0}$ is a twisted AKSZ sigma model defined on the boundary $\partial \mathcal{X}$. In one method, we construct an AKSZ sigma model with a boundary and then reduce the theory to the boundary. In the other method, we reduce a QP pair to a small twisted QP manifold and then construct a twisted AKSZ sigma model. The two methods produce the same twisted AKSZ sigma model $\mathbf{t A K S Z} \mathbf{Z}_{\partial \mathcal{X}, \mathcal{L}}^{0}$.

There is an ambiguity in the definition of an AKSZ sigma model pair because there is an ambiguity in how we should take a big QP manifold for a fixed twisted QP manifold. The cohomology class of $\Theta$ for the big bracket determines the equivalence class of this ambiguity. In the language of sigma models, if $\left.S_{b u l k}\right|_{\partial \mathcal{X}}=0$ for $S_{T^{*}[n+1] \mathcal{L}}^{\prime}=S_{T^{*}[n+1] \mathcal{L}}+S_{\text {bulk }}$ on $\mathcal{X}$, then $S$ and $S^{\prime}$ define the same boundary in the twisted AKSZ sigma models.

We will now show some examples of twisted AKSZ sigma models.

Example 5.1. ( $n=2$ : twisted-(or WZ-)Poisson sigma models). We consider a QP pair for the case of $\Theta_{C}=\Theta_{R}=\rho=0$ in Example 4.1. The big QP manifold is $T^{*}[2] \mathcal{L}=T^{*}[2] T[1] M$, and the QP structure defines the twisted Poisson structure $\left[\pi_{\mathcal{L}}, \pi_{\mathcal{L}}\right]_{S}=\wedge^{3} \pi^{\#} H$.

Let us take a three-dimensional manifold $X$ for which the boundary is a two-dimensional manifold $\partial X$. The AKSZ sigma model is defined on $\operatorname{Map}\left(T[1] X, T^{*}[2] T[1] M\right)$ as follows. The P-structure is

$$
\boldsymbol{\omega}=\int_{\mathcal{X}} \mu\left(\delta \boldsymbol{x}^{i} \wedge \delta \boldsymbol{\xi}_{i}+\delta \boldsymbol{p}_{i} \wedge \delta \boldsymbol{q}^{i}\right),
$$

where the boldface letters are superfields induced from the pullbacks by $\boldsymbol{x}^{*}$ of corresponding local coordinates. If $\alpha=0$, the Q-structure function has the following form:

$$
S=S_{0}+S_{1}=\int_{\mathcal{X}} \mu\left(-\boldsymbol{\xi}_{i} \boldsymbol{d} \boldsymbol{x}^{i}+\boldsymbol{q}^{i} \boldsymbol{d} \boldsymbol{p}_{i}+\boldsymbol{\xi}_{i} \boldsymbol{q}^{i}+\frac{1}{3 !} H_{i j k}(\boldsymbol{x}) \boldsymbol{q}^{i} \boldsymbol{q}^{j} \boldsymbol{q}^{k}\right) .
$$

In order to obtain the equations of motion from the variational principle, we take the variation of $S$,

$$
\delta S=\int_{\mathcal{X}} \mu\left(-\delta \boldsymbol{\xi}_{i} \boldsymbol{d} \boldsymbol{x}^{i}-\boldsymbol{\xi}_{i} \boldsymbol{d} \delta \boldsymbol{x}^{i}+\delta \boldsymbol{q}^{i} \boldsymbol{d} \boldsymbol{p}_{i}+\boldsymbol{q}^{i} \boldsymbol{d} \delta \boldsymbol{p}_{i}+\delta\left(\boldsymbol{\xi}_{i} \boldsymbol{q}^{i}+\frac{1}{3 !} H_{i j k}(\boldsymbol{x}) \boldsymbol{q}^{i} \boldsymbol{q}^{j} \boldsymbol{q}^{k}\right)\right) .
$$

The equations of motion of $\boldsymbol{\xi}$ and $\boldsymbol{q}$ are obtained by integration by parts. Therefore, the boundary terms, $-\boldsymbol{\xi}_{i} \boldsymbol{d} \delta \boldsymbol{x}^{i}+\boldsymbol{q}^{i} \boldsymbol{d} \delta \boldsymbol{p}_{i}$, must vanish:

$$
\left.\delta S\right|_{\partial \mathcal{X}}=\left.\int_{\partial \mathcal{X}} \mu_{\partial \mathcal{X}}\left(-\boldsymbol{\xi}_{i} \delta \boldsymbol{x}^{i}-\boldsymbol{q}^{i} \delta \boldsymbol{p}_{i}\right)\right|_{\partial \mathcal{X}}=0 .
$$

This determines the boundary conditions. Equation (5.8) is satisfied if $\vartheta=0$ on $\operatorname{Im} \partial \mathcal{X}$, i.e., $\operatorname{Im} \partial \mathcal{X} \subset \mathcal{L}$. Typically, two kinds of boundary conditions are possible: $\boldsymbol{\xi}_{/ / i}=0$ or $\delta \boldsymbol{x}_{/ /}^{i}=0$, and $\boldsymbol{q}_{/ /}^{i}=0$ or $\delta \boldsymbol{p}_{/ / i}=0$, where // is the component that is parallel to the boundary. (Hybrid boundary conditions are also possible.)

Let us take boundary conditions $\boldsymbol{\xi}_{/ / i}=0$ and $\boldsymbol{q}_{/ /}^{i}=0$ as an example. Another condition is that the boundary conditions must be consistent with the classical master equation $\{S, S\}=0$. Direct 
computation gives

$$
\{S, S\}=\left.\int_{\partial \mathcal{X}} \mu_{\partial \mathcal{X}}\left(-\boldsymbol{\xi}_{i} \boldsymbol{d} \boldsymbol{x}^{i}+\boldsymbol{q}^{i} \boldsymbol{d} \boldsymbol{p}_{i}+\boldsymbol{\xi}_{i} \boldsymbol{q}^{i}+\frac{1}{3 !} H_{i j k}(\boldsymbol{x}) \boldsymbol{q}^{i} \boldsymbol{q}^{j} \boldsymbol{q}^{k}\right)\right|_{\partial \mathcal{X}} .
$$

Due to the boundary conditions $\boldsymbol{\xi}_{/ / i}=0$ and $\boldsymbol{q}_{/ /}^{i}=0$, the first two terms corresponding to $\vartheta$ of the right-hand side vanish on the boundary:

$$
\int_{\partial \mathcal{X}} \mu_{\partial \mathcal{X}}\left(i_{\partial} \times \mathrm{id}\right)^{*} \mathrm{ev}^{*} \vartheta=\left.\int_{\partial \mathcal{X}} \mu_{\partial \mathcal{X}}\left(-\boldsymbol{\xi}_{i} \boldsymbol{d} \boldsymbol{x}^{i}+\boldsymbol{q}^{i} \boldsymbol{d} \boldsymbol{p}_{i}\right)\right|_{\partial \mathcal{X}}=0 .
$$

Therefore, the last two terms corresponding to the $\Theta$ terms in Eq. (5.9) must vanish:

$$
\int_{\partial \mathcal{X}} \mu_{\partial \mathcal{X}}\left(i_{\partial} \times \mathrm{id}\right)^{*} \mathrm{ev}^{*} \Theta=\left.\int_{\partial \mathcal{X}} \mu_{\partial \mathcal{X}}\left(\boldsymbol{\xi}_{i} \boldsymbol{q}^{i}+\frac{1}{3 !} H_{i j k}(\boldsymbol{x}) \boldsymbol{q}^{i} \boldsymbol{q}^{j} \boldsymbol{q}^{k}\right)\right|_{\partial \mathcal{X}}=0 .
$$

The Q-structure follows immediately from the boundary conditions $\boldsymbol{\xi}_{/ / i}=0$ and $\boldsymbol{q}_{/ /}^{i}=0$.

This consistency of the boundary conditions is described in terms of a target QP manifold $T^{*}[2] \mathcal{L}$. Equation (5.10) is satisfied if $\left.\vartheta\right|_{\mathcal{L}}=0$. Under this condition, Eq. (5.11) is satisfied if $\left.\mathrm{ev}^{*} \Theta\right|_{\partial \mathcal{X}}=0$. This condition is the pullback of the equation $\left.\Theta\right|_{\mathcal{L}}=0$. This corresponds to Proposition 3.2.

Next we introduce $\alpha$. The Q-structure is modified by introducing a canonical function $\alpha$. For example, we take $\int_{\partial \mathcal{X}} \mu_{\partial \mathcal{X}}\left(i_{\partial} \times \mathrm{id}\right)^{*} \mathrm{ev}^{*} \alpha=\int_{\partial \mathcal{X}} \mu_{\partial \mathcal{X}} \frac{1}{2} \pi^{i j}(\boldsymbol{x}) \boldsymbol{p}_{i} \boldsymbol{p}_{j}$. The Q-structure changes to

$$
\begin{aligned}
S^{\prime}= & \int_{\mathcal{X}} \mu\left(-\boldsymbol{\xi}_{i} \boldsymbol{d} \boldsymbol{x}^{i}+\boldsymbol{q}^{i} \boldsymbol{d} \boldsymbol{p}_{i}+\boldsymbol{\xi}_{i} \boldsymbol{q}^{i}+\frac{1}{3 !} H_{i j k}(\boldsymbol{x}) \boldsymbol{q}^{i} \boldsymbol{q}^{j} \boldsymbol{q}^{k}\right) \\
& -\int_{\partial \mathcal{X}} \mu_{\partial \mathcal{X}} \frac{1}{2} \pi^{i j}(\boldsymbol{x}) \boldsymbol{p}_{i} \boldsymbol{p}_{j} .
\end{aligned}
$$

The boundary term deforms the boundary conditions. The variation $\delta S$ is changed to

$$
\left.\delta S^{\prime}\right|_{\partial \mathcal{X}}=\int_{\partial \mathcal{X}} \mu_{\partial \mathcal{X}}\left[\left(-\boldsymbol{\xi}_{i}+\frac{1}{2} \frac{\partial \pi^{i j}(\boldsymbol{x})}{\partial \boldsymbol{x}^{i}} \boldsymbol{p}_{j} \boldsymbol{p}_{k}\right) \delta \boldsymbol{x}^{i}+\left(-\boldsymbol{q}^{i}-\pi^{i j}(\boldsymbol{x}) \boldsymbol{p}_{j}\right) \delta \boldsymbol{p}_{i}+\cdots\right] .
$$

Since these terms must vanish, consistent boundary conditions are as follows:

$$
\left.\boldsymbol{\xi}_{i}\right|_{/ /}=-\left.\frac{1}{2} \frac{\partial \pi^{j k}}{\partial \boldsymbol{x}^{i}}(\boldsymbol{x}) \boldsymbol{p}_{j} \boldsymbol{p}_{k}\right|_{/ /},\left.\quad \boldsymbol{q}^{i}\right|_{/ /}=\left.\pi^{i j}(\boldsymbol{x}) \boldsymbol{p}_{j}\right|_{/ /}
$$

$\left\{S^{\prime}, S^{\prime}\right\}=0$ requires another consistency condition, i.e., the integrand of $S_{1}$ is zero on the boundary:

$$
\left.\left(\boldsymbol{\xi}_{i} \boldsymbol{q}^{i}+\frac{1}{3 !} H_{i j k}(\boldsymbol{x}) \boldsymbol{q}^{i} \boldsymbol{q}^{j} \boldsymbol{q}^{k}\right)\right|_{/ /}=0 .
$$

Similarly, (5.13) and (5.14) can be expressed by the condition on $T^{*}[2] \mathcal{L}=T^{*}[2] T[1] M$. The condition is that

$$
\xi_{i} q^{i}+\frac{1}{3 !} H_{i j k}(x) q^{i} q^{j} q^{k}=0
$$

on the Lagrangian submanifold $\mathcal{L}^{\prime}$ defined by

$$
\xi_{i}=-\frac{1}{2} \frac{\partial \pi^{j k}}{\partial x^{i}}(x) p_{j} p_{k}, \quad q^{i}=\pi^{i j}(x) p_{j}
$$

Here we consider the symplectic form graded by $e^{-\delta_{\alpha}}$ while preserving $Q . \mathcal{L}^{\prime}$ is a Lagrangian submanifold with respect to $e^{-\delta_{\alpha}} \omega$. Substituting (5.16) into (5.15), we obtain the geometric structure on $\mathcal{L}^{\prime}$ :

$$
\begin{aligned}
& \xi_{i} q^{i}+\frac{1}{3 !} H_{i j k}(x) q^{i} q^{j} q^{k} \\
& =-\frac{1}{2} \frac{\partial \pi^{j k}}{\partial x^{l}}(x) \pi^{l i}(x) p_{j} p_{k} p_{i}+\frac{1}{3 !} H_{i j k}(x) \pi^{i l}(x) \pi^{j m}(x) \pi^{k n}(x) p_{l} p_{m} p_{n}=0 .
\end{aligned}
$$


This condition corresponds to Proposition 3.5. Equation (5.17) is nothing but the equation of the twisted Poisson structure $[\pi, \pi]_{S}=\wedge^{3} \pi^{\#} H$.

Physically, the boundary action on $\partial T[1] X$ is obtained by integrating out the superfield $\xi_{i}$ from Eq. (5.12). By integrating out $\boldsymbol{\xi}_{i}$, we obtain the equations of motion $\boldsymbol{q}^{i}=\boldsymbol{d} \boldsymbol{x}^{i}$. By restricting this orbit, we obtain the twisted-Poisson sigma model on $\mathcal{L}^{\prime}$ :

$$
S_{\mathcal{L}^{\prime}}=\int_{\partial \mathcal{X}} \mu_{\partial \mathcal{X}}\left(\boldsymbol{p}_{i} \boldsymbol{d} \boldsymbol{x}^{i}-\frac{1}{2} \pi^{i j}(\boldsymbol{x}) \boldsymbol{p}_{i} \boldsymbol{p}_{j}\right)+\int_{\mathcal{X}} \mu_{\mathcal{X}} \frac{1}{3 !} H_{i j k}(\boldsymbol{x}) \boldsymbol{d} \boldsymbol{x}^{i} \boldsymbol{d} \boldsymbol{x}^{j} \boldsymbol{d} \boldsymbol{x}^{k} .
$$

This coincides with the physical model constructed from the twisted Poisson structure on $\partial X,{ }^{29}$ and it is the twisted AKSZ sigma model constructed from the twisted Poisson structure on $\mathcal{L}^{\prime}$. The Pstructure on $\operatorname{Map}\left(T[1] X, \mathcal{L}^{\prime}\right)$ is equivalent to the one constructed from the derived bracket $\{-,-\}_{\Theta}=$ $\left.\{\{-, \Theta\},-\}\right|_{\operatorname{Map}\left(\partial \mathcal{X}, \mathcal{L}^{\prime}\right)}$. The $H$ term breaks the classical master equation, i.e., $\left\{S_{\mathcal{L}^{\prime}}, S_{\mathcal{L}^{\prime}}\right\}_{\Theta} \neq 0$.

Example 5.2. ( $n=3$ : the twisted strong Courant sigma models). Let us consider the AKSZ sigma model induced from a QP manifold of degree 3 and its canonical function. We take a QP manifold of degree $3, T^{*}[3] \mathcal{L}=T^{*}[3]\left(T^{*}[2] E[1] \oplus A[2]\right)$, as a generalization of Sec. IV C, where $E$ and $A$ are two vector bundles on $M$.

We take the local coordinates on $T^{*}[2] E[1] \oplus A[2]$ as $\left(x^{i}, u^{a}, z_{p}, p_{i}\right)$ of degree $(0,1,2,2)$, where $x^{i}$ is a local coordinate on $M, u^{a}$ is on the fiber of $E, z_{p}$ is on the fiber of $A$, and $p_{i}$ is on the fiber of $T^{*}[2] M$. The conjugate local coordinates of the fiber are $\left(\xi_{i}, v_{a}, w^{p}, q^{i}\right)$ of degree $(3,2,1,1)$. A graded symplectic structure is given by $\omega=\delta x^{i} \wedge \delta \xi_{i}-\delta u^{a} \wedge \delta v_{a}+\delta p_{i} \wedge \delta q^{i}+\delta z_{p} \wedge \delta w^{p}$. We consider the following Q-structure satisfying $\left.\Theta\right|_{\mathcal{L}}=0$ :

$$
\Theta=\xi_{i} q^{i}+\frac{1}{2} k^{a b} v_{a} v_{b}+\rho_{r}^{i}(x) \xi_{i} w^{r}+\frac{1}{2} C^{r}{ }_{p q}(x) z_{r} w^{p} w^{q}+\frac{1}{4 !} H_{i j k l}(x) q^{i} q^{j} q^{k} q^{l},
$$

where $k^{a b}$ is a fiber metric on $E$ and $H=\frac{1}{4 !} H_{i j k l}(x) d x^{i} \wedge d x^{j} \wedge d x^{k} \wedge d x^{l}$ is a 4-form on $M$. If $A$ is a Lie algebroid and $H$ is closed, $\{\Theta, \Theta\}=0$ is satisfied.

Take the Lagrangian submanifold $\mathcal{L}=T^{*}[2] E[1] \oplus A[2]=\left\{\xi_{i}=v_{a}=q^{i}=w^{p}=0\right\}$ and a function of degree 3 ,

$$
\alpha=\sigma_{a}^{i}(x) p_{i} u^{a}+\tau^{r}{ }_{a}(x) z_{r} u^{a}+\frac{1}{3 !} h_{a b c}(x) u^{a} u^{b} u^{c} .
$$

The canonical function equation $\left.e^{\delta_{\alpha}} \Theta\right|_{\mathcal{L}}=0$ determines the identities among $\sigma_{a}^{i}(x), \tau^{r}{ }_{a}(x)$, and $h_{a b c}(x)$ and the geometric conditions on $\mathcal{L}$.

If $w^{p}=z_{p}=0$ and $H=0$, this reduces to the Courant algebroid. If $w^{p}=z_{p}=0$ and $H \neq 0$, this reduces to the $H_{4}$-twisted Courant algebroid in Example 4.2. If $q^{i}=p_{i}=0$, this reduces to the strong Courant algebroid.

Let us take a four-dimensional manifold $X$ with a boundary that is a three-dimensional manifold $\partial X$. The bulk AKSZ sigma model on $T^{*}[3]\left(T^{*}[2] E[1] \oplus A[1]\right)$ is constructed in the usual way. The P-structure is

$$
\boldsymbol{\omega}=\int_{\mathcal{X}} \mu\left(\delta \boldsymbol{x}^{i} \wedge \delta \boldsymbol{\xi}_{i}+\delta \boldsymbol{p}_{i} \wedge \delta \boldsymbol{q}^{i}-\delta \boldsymbol{u}^{a} \wedge \delta \boldsymbol{v}_{a}+\delta \boldsymbol{z}_{p} \wedge \delta \boldsymbol{w}^{p}\right) .
$$

The Q-structure function with boundary terms has the following form:

$$
\begin{aligned}
S= & \int_{\mathcal{X}} \mu\left(\boldsymbol{\xi}_{i} \boldsymbol{d} \boldsymbol{x}^{i}+\boldsymbol{q}^{i} \boldsymbol{d} \boldsymbol{p}_{i}-\boldsymbol{v}_{a} \boldsymbol{d} \boldsymbol{u}^{a}+\boldsymbol{w}^{p} \boldsymbol{d} z_{p}+\boldsymbol{\xi}_{i} \boldsymbol{q}^{i}\right. \\
& \left.+\frac{1}{2} k^{a b} \boldsymbol{v}_{a} \boldsymbol{v}_{b}+\rho^{i}{ }_{r}(\boldsymbol{x}) \boldsymbol{\xi}_{i} \boldsymbol{w}^{r}+\frac{1}{2} C^{r}{ }_{p q}(\boldsymbol{x}) \boldsymbol{z}_{r} \boldsymbol{w}^{p} \boldsymbol{w}^{q}+\frac{1}{4 !} H_{i j k l}(\boldsymbol{x}) \boldsymbol{q}^{i} \boldsymbol{q}^{j} \boldsymbol{q}^{k} \boldsymbol{q}^{l}\right) \\
& -\int_{\partial \mathcal{X}} \mu_{\partial X}\left(\sigma^{i}{ }_{a}(\boldsymbol{x}) \boldsymbol{p}_{i} \boldsymbol{u}^{a}+\tau^{r}{ }_{a}(\boldsymbol{x}) \boldsymbol{z}_{r} \boldsymbol{u}^{a}+\frac{1}{3 !} h_{a b c}(\boldsymbol{x}) \boldsymbol{u}^{a} \boldsymbol{u}^{b} \boldsymbol{u}^{c}\right) .
\end{aligned}
$$

We can construct the boundary of the twisted AKSZ sigma model by using the method in this section. In this example, the derived Poisson bracket on $\mathcal{L}$ is generally degenerate. In degenerate cases, it is 
difficult to express concretely the boundary Q-structure function $S$. However, the bulk and boundary theories are physically consistent because the bulk classical master equation is satisfied.

If $A=0$, the QP manifold is $T^{*}[3] \mathcal{L}=T^{*}[3] T^{*}[2] E[1]$, and the derived Poisson bracket is nondegenerate. The corresponding twisted QP manifold $T^{*}[2] E[1]$ produces the boundary topological sigma model with a Wess-Zumino term from a three-dimensional manifold $\partial X$ to the target space $T^{*}[2] E[1]:$

$$
\begin{aligned}
S_{\mathcal{L}}= & \int_{\partial \mathcal{X}} \mu_{\partial \mathcal{X}}\left(\boldsymbol{p}_{i} \boldsymbol{d} \boldsymbol{x}^{i}-\frac{1}{2} k_{a b} \boldsymbol{u}^{a} \boldsymbol{d} \boldsymbol{u}^{b}-\sigma_{a}^{i}(\boldsymbol{x}) \boldsymbol{p}_{i} \boldsymbol{u}^{a}-\frac{1}{3 !} h_{a b c}(x) \boldsymbol{u}^{a} \boldsymbol{u}^{b} \boldsymbol{u}^{c}\right) \\
& +\int_{\mathcal{X}} \mu \frac{1}{4 !} H_{i j k l}(\boldsymbol{x}) \boldsymbol{d} \boldsymbol{x}^{i} \boldsymbol{d} \boldsymbol{x}^{j} \boldsymbol{d} \boldsymbol{x}^{k} \boldsymbol{d} \boldsymbol{x}^{l} .
\end{aligned}
$$

This twisted Q-structure function can also be obtained by integrating out $\boldsymbol{\xi}_{i}$ and $\boldsymbol{v}_{a}$ in Eq. (5.20) by physical arguments. This is equivalent to the $H_{4}$-twisted Courant sigma model. ${ }^{20}$

\section{SUMMARY AND FUTURE AREAS OF WORK}

In this paper, we have analyzed the mathematical structures of the boundary conditions of AKSZ sigma models and boundary theories, and we have discussed the connections between canonical functions and QP pairs. We have proposed a new algebroid, the strong Courant algebroid.

However, we have not completely analyzed the properties of the solutions of the canonical function equations, the deformation theory, and the equivalence classes of the QP description of a twisted QP manifold. The deformation theory of QP structures will unify various classical and new structures, for example, the Nijenhuis structures on Lie algebras, the Courant-like algebroid structures in Poisson geometry, and others. ${ }^{31}$ In order to understand the complete structures, we should consider the general classification of a QP pair $\left(T^{*}[n+1] \mathcal{L}, \omega, \Theta, \mathcal{L}, \alpha\right)$ and $\left(\mathcal{L}, \omega_{\mathcal{L}}, \alpha_{\mathcal{L}}\right)$, which can be viewed as a simultaneous deformation of the homological function $\Theta$ and the canonical function $\alpha$.

In this paper, all of the examples derived from canonical functions are geometric. If we consider a QP manifold and a canonical function over a point, we can derive other kinds of algebraic structures. We will leave these as areas to investigate in future work.

The quantization of AKSZ sigma models with boundaries is the next step. Since AKSZ sigma models are topological field theories, a bulk AKSZ sigma model and the corresponding boundary theory should have the same physical partition functions and produce equivalent mathematical and physical information. This will not only describe the quantum membrane theories in physics but will also quantize a wide class of geometric structures.

\section{ACKNOWLEDGMENTS}

The authors would like to thank J. Stasheff for valuable discussions and comments. N. Ikeda would like to thank the organizers of The 33rd Winter School Geometry and Physics, Srni, Czech Republic, where part of this work was performed. X.-M. Xu is grateful to Zhangju Liu for his patient guidance throughout his study at Peking University. He also would like to thank Jianghua Lu for hospitality during his visit to the University of Hong Kong, where part of this work was performed. X.-M. Xu is supported by Grant No. PDFMP2-141756 of the Swiss National Science Foundation.

${ }^{1}$ A. Alekseev, Y. Kosmann-Schwarzbach, and E. Meinrenken, "Quasi-Poisson manifolds," Canadian J. Math. 54, 3-29 (2002); e-print arXiv:math/0006168.

${ }^{2}$ M. Alexandrov, M. Kontsevich, A. Schwartz, and O. Zaboronsky, "The geometry of the master equation and topological quantum field theory,” Int. J. Mod. Phys. A 12, 1405 (1997); e-print arXiv:hep-th/9502010.

${ }^{3}$ R. Bousso, "The holographic principle, Rev. Mod. Phys. 74, 825-874 (2002).

${ }^{4}$ P. Bouwknegt and B. Jurčo, "AKSZ construction of topological open p-brane action and Nambu brackets," Rev. Math. Phys. 25, 1330004 (2013); e-print arXiv:1110.0134 [math-ph].

${ }^{5}$ D. Calaque, "Lagrangian structures on mapping stacks and semi-classical TFTs," e-print arXiv:1306.3235 [math.AG] (unpublished). 
${ }^{6}$ C. Carmeli, L. Caston, and R. Fioresi, "Mathematical foundation of supersymmetry," in EMS Series of Lectures in Mathematics (European Mathematical Society, Zurich, 2011).

${ }^{7}$ A. S. Cattaneo and G. Felder, "A path integral approach to the Kontsevich quantization formula," Commun. Math. Phys. 212, 591 (2000); e-print arXiv:math/9902090.

${ }^{8}$ A. S. Cattaneo and G. Felder, "On the AKSZ formulation of the Poisson sigma model," Lett. Math. Phys. 56, 163 (2001); e-print arXiv:math/0102108.

${ }^{9}$ A. Cattaneo, P. Mnev, and N. Reshetikhin, "Classical BV theories on manifolds with boundary," Commun. Math. Phys. 332, 535 (2014).

${ }^{10}$ A. S. Cattaneo, P. Mnev, and N. Reshetikhin, "Classical and quantum Lagrangian field theories with boundary," PoS CORFU 2011, 044; e-print arXiv:1207.0239 [math-ph].

${ }^{11}$ A. S. Cattaneo and F. Schätz, "Introduction to supergeometry," Rev. Math. Phys. 23, 669-690 (2011)

${ }^{12}$ T. Courant, "Dirac manifolds," Trans. A. M. S. 319, 631 (1990).

${ }^{13}$ I. Ya. Dorfman, "Dirac structures of integrable evolution equations," Phys. Lett. A 125, 240-246 (1987).

${ }^{14}$ R. L. Fernandes, "Lie algebroids, holonomy, and characteristic class," Adv. Math. 170, 119-179 (2002).

${ }^{15}$ D. Fiorenza, C. L. Rogers, and U. Schreiber, "A higher Chern-Weil derivation of AKSZ $\sigma$-models," Int. J. Geom. Meth. Mod. Phys. 10, 1250078 (2013); e-print arXiv: 1108.4378 [math-ph].

${ }^{16}$ D. Fiorenza, H. Sati, and U. Schreiber, "Super Lie n-algebra extensions, higher WZW models, and super p-branes with tensor multiplet fields," e-print arXiv:1308.5264 [hep-th] (unpublished).

${ }^{17}$ Y. Fregier and M. Zambon, "Simultaneous deformations and Poisson geometry," e-print arXiv:1202.2896 [math.QA] (unpublished).

${ }^{18}$ Y. Fregier and M. Zambon, "Simultaneous deformations of algebras and morphisms via derived brackets," e-print arXiv:1301.4864 [math.QA] (unpublished).

${ }^{19}$ M. Grützmann, "H-twisted Lie algebroid,” J. Geom. Phys. 61, 476 (2011); e-print arXiv:1005.5680.

${ }^{20}$ M. Hansen and T. Strobl, "First class constrained systems and twisting of Courant algebroids by a closed 4-form," e-print arXiv:0904.0711 [hep-th] Fundamental interactions, 115-144, World Sci. Publ., Hackensack, NJ, 2010.

${ }^{21}$ H. Hata and B. Zwiebach, "Developing the covariant Batalin-Vilkovisky approach to string theory," Ann. Phys. 229, 177 (1994); e-print arXiv:hep-th/9301097.

${ }^{22}$ C. Hofman and J. S. Park, “Topological open membranes," e-print arXiv:hep-th/0209148 (unpublished).

${ }^{23} \mathrm{~N}$. Ikeda, "Two-dimensional gravity and nonlinear gauge theory," Annals Phys. 235, 435 (1994); e-print arXiv:hep-th/9312059.

${ }^{24}$ N. Ikeda, "Chern-Simons gauge theory coupled with BF theory," Int. J. Mod. Phys. A 18, 2689 (2003); e-print arXiv:hep-th/0203043.

${ }^{25}$ N. Ikeda, "Lectures on AKSZ topological field theories for physicists," e-print arXiv:1204.3714 [hep-th] (unpublished).

${ }^{26}$ N. Ikeda and K. I. Izawa, "General form of dilaton gravity and nonlinear gauge theory," Prog. Theor. Phys. 90, 237 (1993); e-print arXiv:hep-th/9304012.

${ }^{27}$ N. Ikeda and K. Uchino, "QP-Structures of degree 3 and 4D topological field theory," Commun. Math. Phys. 303, 317 (2011); e-print arXiv:1004.0601 [hep-th].

${ }^{28}$ N. Ikeda and X.-M. Xu, "Current algebras from DG symplectic pairs in supergeometry,” e-print arXiv:1308.0100 [math-ph] (unpublished).

${ }^{29}$ C. Klimcik and T. Strobl, "WZW-Poisson manifolds," J. Geom. Phys. 43, 341 (2002); e-print arXiv:math/0104189.

${ }^{30}$ M. Kontsevich, "Deformation quantization of Poisson manifolds," Lett. Math. Phys. 66, 157 (2003); e-print arXiv:q-alg/9709040.

${ }^{31}$ Y. Kosmann-Schwarzbach and F. Magri, "Poisson-Nijenhuis structures," Ann. Inst. Henri Poincare, Serie A 53, 35-81 (1990).

${ }^{32}$ Y. Kosmann-Schwarzbach, "Derived brackets," Lett. Math. Phys. 69, 61 (2004); e-print arXiv:math.dg/0312524.

${ }^{33}$ Y. Kosmann-Schwarzbach, "Poisson and symplectic functions in Lie algebroid theory," in Higher Structures in Geometry and Physics, in honor of Murray Gerstenhaber and Jim Stashef, Progress in Mathematics Vol. 287, edited by Alberto Cattaneo, Antony Giaquinto, and Ping Xu (Birkhauser, 2011), pp. 243-268; e-print arXiv:0711.2043.

${ }^{34}$ H.-L. Lang, Y.-H. Sheng, and X.-M. Xu, "Graded Poisson manifolds up to homotopy," e-print arXiv:1312.4096 (unpublished).

${ }^{35}$ Z.-J. Liu, A. Weinstein, and P. Xu, "Manin triples for Lie bialgebroids," J. Diff. Geom. 45, 547-574 (1997).

${ }^{36}$ J.-H. Lu, "Poisson homogeneous spaces and Lie algebroids associated to Poisson actions," Duke Math. J. 86, 261-304 (1997).

${ }^{37}$ T. Pantev, B. Toen, M. Vaquie, and G. Vezzosi, "Shifted symplectic structures," Publications mathématiques de l'IHÉS 117, 271-328 (2013); e-print arXiv:1111.3209 [math.AG].

${ }^{38}$ J. S. Park, "Topological open p-branes," in Symplectic Geometry and Mirror Symmetry, edited by K. Fukaya, Y.-G. Oh, K. Ono, and G. Tian (World Scientific, 2001), pp. 311-384.

${ }^{39}$ D. Roytenberg, "Courant algebroids, derived brackets and even symplectic supermanifolds," e-print arXiv:math.DG/9910078 (unpublished).

${ }^{40}$ D. Roytenberg, "Quasi Lie bialgebroids and twisted Poisson manifolds," Lett. Math. Phys. 61, 123 (2002); e-print arXiv:math/0112152 [math-qa].

${ }^{41}$ D. Roytenberg, "AKSZ-BV formalism and Courant algebroid-induced topological field theories," Lett. Math. Phys. 79, 143 (2007); e-print arXiv:hep-th/0608150.

${ }^{42}$ P. Schaller and T. Strobl, "Poisson structure induced (topological) field theories," Mod. Phys. Lett. A 9, 3129 (1994); e-print arXiv:hep-th/9405110.

${ }^{43}$ A. Schwarz, "Geometry of Batalin-Vilkovisky quantization," Commun. Math. Phys. 155, 249 (1993), e-print arXiv:hep-th/9205088; "Semiclassical approximation in Batalin-Vilkovisky formalism," ibid. 158, 373 (1993); e-print arXiv:hep-th/9210115. 
${ }^{44}$ P. Ševera and A. Weinstein, "Poisson geometry with a 3 form background," Prog. Theor. Phys. Suppl. 144, 145 (2001); e-print arXiv:math/0107133 [math-sg].

${ }^{45}$ Y. Terashima, "On Poisson functions," J. Sympl. Geom. 6, 1-7 (2008).

${ }^{46}$ V. S. Varadarajan, "Supersymmetry for mathematicians: An introduction," Courant Lecture Notes Series (AMS, New York, 2004).

${ }^{47}$ A. Vaintrob, "Lie algebroids and homological vector fields," Uspekhi Mat. Nauk 52(2(314)), 161-162 (1997) [Russian Math. Surveys 52(2), 428-429 (1997)].

${ }^{48}$ T. Voronov, "Graded manifolds and drinfeld doubles for Lie bialgebroids," in Quantization, Poisson Brackets and Beyond, Contemporary Mathematics Vol. 315, edited by Theodore Voronov (Amer. Math. Soc., Providence, RI, 2002), pp. 131-168; e-print arXiv:math/0105237 [math-dg].

${ }^{49}$ X.-M. Xu, "Twisted Courant algebroids and coisotropic Cartan geometries," J. Geom. Phys. 82, 124-131 (2014). 